\title{
Perspectives and Consensus among International Orthopaedic Surgeons during Initial and Mid-lockdown Phases of Coronavirus Disease
}

J. Terrence Jose Jerome ${ }^{1}$ Francisco Mercier ${ }^{2}$ Chaitanya S. Mudgal ${ }^{3,4} \quad$ Joan Arenas-Prat ${ }^{5}$ Gustavo Vinagre ${ }^{6}$ Chul Ki Goorens $^{7}$ Ignacio J. Rivera-Chavarría ${ }^{8}$ Sreedharan Sechachalam ${ }^{9}$ Bolaji Mofikoya ${ }^{10}$ Achilleas Thoma $^{11}$ Claudia Medina ${ }^{12} \quad$ IlavarasuTamilmani ${ }^{13}$ Ignacio J. Rivera-Chavarría ${ }^{14}$ Mark Henry ${ }^{15}$ Ahmadreza Afshar ${ }^{16}$ Zoe H. Dailiana ${ }^{17}$ Theddeus O.H. Prasetyono ${ }^{18}$ Stefano Artiaco ${ }^{19}$ Thayur R Madhusudhan ${ }^{20}$ Skender Ukaj21 ${ }^{21}$ Ole Reigstad ${ }^{22}$ Yoshitaka Hamada ${ }^{23}$ Rajesh Bedi24 Andrea Poggetti ${ }^{25}$ Mohammad Manna Al-Qattan ${ }^{26}$ Mahdi Siala ${ }^{27}$ Anand Viswanathan ${ }^{28}$ Rafael Romero-Reveron ${ }^{29}$ Joon Pio Hong ${ }^{30}$ Kamarul Ariffin Khalid ${ }^{31}$ Shivashankar Bhaskaran ${ }^{32}$ Krishnamoorthy Venkatadass ${ }^{33}$ Somsak Leechavengvongs ${ }^{34} \quad$ Chul Ki Goorens $^{35,36} \quad$ Sifi Nazim ${ }^{37}$ Alexandru Valentin Georgescu ${ }^{38}$ Mathias Tremp ${ }^{39}$ Kiran K. Nakarmi ${ }^{40} \quad$ Mohamed A. Ellabban ${ }^{41}$ Pingtak Chan ${ }^{42}$ Andrey Aristov ${ }^{43}$ Sandeep Patel ${ }^{44}$ Constanza L. Moreno-Serrano ${ }^{45}$ Shwetabh Rai ${ }^{46}$ Rishi Mugesh Kanna ${ }^{47}$ Vijay A Malshikare ${ }^{48} \quad$ Katsuhisa Tanabe $^{49} \quad$ Simon Thomas $^{50} \quad$ Kemal Gokkus $^{51}$ Seung-Hoon Baek ${ }^{52}$ Jerker Brandt ${ }^{53} \quad$ Yin Rith $^{54} \quad$ Alfredo Olazabal $^{55} \quad$ Muhammad Saaiq $^{56}$ Vijay Patil ${ }^{57}$ $\mathrm{N}$ jithendran ${ }^{58}$ Harshil Parekh ${ }^{59}$ Yoshitaka Minamikawa ${ }^{60} \quad$ Abdulljawad Almabrouk Atagawi $^{61}$ Jalal Ahmed Hadi62 Claudia Arroyo Berezowsky ${ }^{63}$ Joaquin Moya-Angeler ${ }^{64}$ Marco Antonio Altamirano-Cruz ${ }^{65}$ Luz Adriana Galvis R $R^{66} \quad$ Alex Antezana ${ }^{67} \quad$ Lukasz Paczesny ${ }^{68} \quad$ Carlos Henrique Fernandes $^{69} \quad$ Md. Asadullah $^{70}$ Lo Yuan-Shun $^{71}$ Biser Makelov ${ }^{72}$ Chaitanya Dodakundi ${ }^{73}$ Rabindra Regmi ${ }^{74}$ Ganarlo Urquizo Pereira ${ }^{75}$ Shuwei Zhang ${ }^{76}$ Binoy Sayoojianadhan ${ }^{77}$ Ivan Callupe ${ }^{78} \quad$ Mohamed I. Rakha $^{79}$ Dino Papes ${ }^{80}$ Ramesh Prabu Ganesan ${ }^{81}$ Mukesh Mohan ${ }^{82}$ Arun Jeyaraman ${ }^{83}$ Ponnaian Prabhakar ${ }^{84}$ Arungeethayan Rajniashokan ${ }^{85} \quad$ I. Geethan ${ }^{86}$ Sugavanam Chandrasekar ${ }^{87}$ Steffen Löw ${ }^{88}$ Kannan Thangavelu ${ }^{89} \quad$ Luca Dei Giudici $^{90}$ Yuvarajan Palanisamy ${ }^{91} \quad$ Singaravadivelu Vaidyanathan ${ }^{92}$ Jorge Boretto $^{93}$ Monica Alexandra Ramirez ${ }^{94}$ Thirumalaisamy Subbiah Goundar ${ }^{95}$ Thirumavalavan Kuppusamy ${ }^{96}$ Kalaivanan Kanniyan ${ }^{97}$ Atul Srivastava ${ }^{98} \quad$ Yung-Cheng Chiu $^{36}$ Anil K Bhat ${ }^{99}$ Nalli R Gopinath ${ }^{100}$ Vijayaraghavan P. Vasudevan ${ }^{101}$ Vineet Abraham ${ }^{102}$

\footnotetext{
${ }^{1}$ Department of Orthopedics, Hand and Reconstructive Microsurgery, Olympia Hospital and Research Centre, Tamil Nadu, India

${ }^{2}$ Clínica Lambert Rua, Cordeiro Ferreira, Lisbon, Portugal

${ }^{3}$ Harvard Medical School, Massachusetts, United States; Hand Surgery Services, Massachusetts General Hospital, Boston, Massachusetts, United States

${ }^{4}$ Hand Surgery Service, Massachusetts General Hospital, Boston, Massachusetts, United States

${ }^{5}$ Department of Orthopaedics, ServeisMedics Penedes, Barcelona, Catalonia, Spain

${ }^{6}$ Department of Orthopaedic Surgery, Aspetar Orthopaedic and Sports Medicine Hospital, Doha, Qatar

${ }^{7}$ Department of Orthopaedics, Regional Hospital Tienen, Tienen, Belgium

${ }^{8}$ Servicio Vascular Periférico, Hospital Rafael Ángel Calderón Guardia, San José, Costa Rica

9 Jalan Tan Tock Seng, Singapore

${ }^{10}$ Department of Surgery, College of Medicine, University of Lagos, Lagos, Nigeria

${ }^{11}$ Department of Orthopaedics, Hamilton, Ontario, Canada
}

Address for correspondence J. Terrence Jose Jerome, FRCS(G)DNB, FNB, European Diploma Hand Surgery, Department of Orthopedics, Hand and Reconstructive Microsurgery, Olympia Hospital and Research Centre, 47, 47A Puthur High Road, Puthur, Trichy 620017, Tamil Nadu, India (e-mail: terrencejose@gmail.com).

12Department of Orthopaedics, Calle, Colombia

${ }^{13}$ Department of Orthopaedics, United Arab Emirates

${ }^{14}$ Servicio Vascular Periférico, Hospital Rafael Ángel Calderón Guardia, San José, Costa Rica

15 Houston, Texas, United States

${ }^{16}$ Department of Orthopedics, Imam Khomeini hospital, Urmia

University of Medical Sciences, Urmia, Iran

${ }^{17}$ Department of Orthopaedic Surgery, Faculty of Medicine, University of Thessaly, Larissa, Greece

${ }^{18}$ Department of Surgery, Cipto Mangunkusumo Hospital, Faculty of Medicine, Universitas Indonesia, Jakarta, Indonesia

${ }^{19}$ Orthopaedic and Trauma Center, CTO, Turin, Italy

${ }^{20} \mathrm{Glan}$ Clwyd Hospital, Bodelwyddan, North wales, United Kingdom

${ }^{21}$ Veternik, Prishtine, Republic of Kosovo

${ }^{22}$ Hand Surgery Unit, Oslo University Hospital, Oslo, Norway 
${ }^{23}$ Hand Surgery Unit, Kansai Medical University Medical Center, Moriguchi City, Osaka, Japan

${ }^{24}$ Fourth Avenue Denistone, Australia

${ }^{25} \mathrm{Hand}$ and Reconstructive Microsurgery Unit, AOU Careggi, Florence, Italy

${ }^{26}$ Riyadh, Saudi Arabia

27 Service d'Orthopedie, chu de purpan, Toulouse, France

28 United Kingdom

${ }^{29}$ Trauma and Orthopaedic Departamento, Centro Médico Docente La Trinidad, Caracas, Venezuela

30 Hand and Reconstructive Microsurgery Unit, Asan Medical Center, Songpagu Seoul, Korea

${ }^{31}$ Department of Orthopedics, IIUM Medical Centre, Jalan Sultan

Ahmad Shah, Kuantan, Pahang, Malaysia

32 Iyer Orthopaedic Centre, Solapur, Maharashtra, India

${ }^{33}$ Department of Pediatric Orthopaedics, India

${ }^{34}$ Department of Orthopaedics, Vichaiyut Hospital, Bangkok,

Thailand

${ }^{35}$ School of Medicine, China Medical University, Taichung, Taiwan

36Department of Orthopedic Surgery, China Medical University Hospital, Taichung, Taiwan

${ }^{37}$ Department of Orthopaedics, Algiers Faculty of Medicine, Algiers, Algeria

${ }^{38}$ Clinical Hospital for Rehabilitation, University of Medicine Iuliu Hatieganu, Cluj Napoca, Cluj-Napoca, Romania

${ }^{39}$ Department of Orthopaedics, Dorfplatz 1, Cham, Switzerland

40 Kirtipur Hospital, Kathmandu, Nepal

${ }^{41}$ Plastic and Reconstructive Surgery Unit, Department of Surgery, Faculty of Medicine, Suez Canal University, Egypt

${ }^{42}$ Department of Orthopaedics and Traumatology, Tuen Mun Hospital, Tuen Mun, Hong Kong

${ }^{43}$ Department of Orthopaedics, Krasnodar, Russia

${ }^{44}$ Hamilton, New Zealand

${ }^{45}$ Bogota, Colombia

46 Madhaw Market Lanka, Uttar Pradesh, India

${ }^{47}$ Coimbatore, India

4818.52 North Hand and Wrist Hospital, Pune, Maharashtra, India

${ }^{49}$ Department of Orthopaedics, Nishinomiya Municipal Central Hospital, Hayashidacho, Nishinomiya, Japan

${ }^{50}$ Department of Orthopaedics, Rohini, Delhi, India

${ }^{51}$ Alanya Research and Practice Center, Baskent University School of Medicine, Saray Mah, Antalya, Turkey

${ }^{52}$ Department of Orthopedic Surgery, School of Medicine, Kyungpook National University, Kyungpook National University Hospital, Daegu, Republic of Korea

53 HandCenter, Öresund, Baltzarsgatan Malmö, Sweden

${ }^{54}$ Department of Orthopaedics, Cambodia

${ }^{55}$ Department of Orthopaedics, CABA, Buenos Aires, Argentina

${ }^{56}$ Department of Hand Surgery, National Institute of Rehabilitation Medicine (NIRM), Islamabad, Pakistan

${ }^{57}$ Orthopedics, Hand and Microsurgery Unit, Basildon University Hospital, Basildon, Essex, United Kingdom

58 Bommanhalli, Bangalore, Karnataka, India

${ }^{59}$ Orthopaedic Speciality Hospital, Rajkot, Gujarat, India

${ }^{60}$ Hand Unit, Namba Hand Centre, Osaka, Japan

${ }^{61}$ GhotAlshaal, Tripoli, Libya

62Department of Orthopaedics, Amman, Jordan
${ }^{63}$ Av. Vasco De Quiroga, México

${ }^{64}$ Department of Orthopaedics, Murcia, Spain

65 Department of Orthopaedics, Mexico, Spain

${ }^{66}$ Bogota, Santa Barbara, Mexico

${ }^{67}$ Calle Tomasbfrias, Boliva

${ }^{68}$ Orvit Clinic, Citomed Healthcare Center, Sklodowskiej, Torun, Poland

${ }^{69}$ Avenida Leoncio de Magalhaes, Sao Paulo, Brazil

${ }^{70}$ Orthopedics and Hand surgery Unit, Eman Medical College Hospital, Savar, Dhaka, Bangladesh

${ }^{71}$ Taiwan, China

${ }^{72}$ Department of Orthopaedics, Stara Zagora, Bulgaria

${ }^{73}$ Department of Orthopaedics, Rashid Hospital, Dubai, United Arab Emirates

${ }^{74}$ Department of Orthopedics and Reconstructive Microsurgery, National Trauma Centre, Kathmandu, Nepal

75 Urbcapillune s/n San Francisco Moquegua, Peru

76 Department of Spine and Bone Tumor Surgery, Zhongnan Hospital of Wuhan University, Wuhan, China

${ }^{77}$ Hand and Reconstructive Microsurgery Unit, Department of Orthopedic Surgery, St James Hospital, Chalakudy, Kerala, India

${ }^{78}$ Avenida Sanchez Carrion, Lima, Peru

${ }^{79}$ Orthopedic Department, Suez Canal university hospital, Ismailia, Egypt

${ }^{80}$ Department of surgery (Ped and Vasc), University Hospital Center Zagreb, Zagreb, Croatia

${ }^{81}$ Department of Orthopedics, KAP Viswanatham Government Medical College, Trichy, India

${ }^{82}$ Trichy, Tamil Nadu, India

832 Chandraganthi Nagar, Bypass Road, Madurai. India

${ }^{84}$ Orthopaedics and Joint Replacement Care Hospitals, Nampally Hyderabad, India

852A Jeyam Paradise, State Bank Officers Colony, Cantonment, Trichy, India

${ }^{86}$ Trichy, Tamil Nadu, India

${ }^{87}$ Salem, Tamil Nadu, India

${ }^{88}$ Clinic for Trauma and Hand Surgery, Bad Mergentheim, Germany

${ }^{89}$ Radha Medical Centre, Erode, Tamil Nadu, India

${ }^{90}$ Ortopedia e Traumatologia, Chirurgiaarticolare di Spalla e Ginocchio, Albodei Medici e Chirurghi di Latina, Cagliari, Italy

${ }^{91}$ Ortho One Orthopedic Speciality Hospital, Coimbatore, Tamil Nadu, India

92Department of Orthopaedics and Traumatology, Madras Medical College, Chennai, Tamil Nadu, India

${ }^{93}$ Ciudad Autonoma de Buenos Aires, Argentina

94 Bucaramanga, Santander, Colombia

${ }^{95}$ Department of Orthopedics, Joint Replacement Surgery Unit, KMCH Hospitals, Coimbatore, Tamil Nadu, India

${ }^{96}$ Department of Orthopedics, Trauma and Joint Replacement, Shri Bharani Hospital, Villupuram, Tamil Nadu, India

${ }^{97} \mathrm{AJRI}$, SIMS Hospitals No 1, Chennai, Tamil Nadu, India

98 Agra, Uttar Pradesh, India

${ }^{99}$ Department of Orthopaedics, KMC, Manipal, Manipal Academy of Higher Education, Manipal, Karnataka, India

${ }^{100}$ Department of Orthopedics, Thoothukudi Medical College, India

${ }^{101}$ Department of Orthopaedics, SRM Medical College, Chennai, Tamil Nadu, India

102Department of Orthopaedics, Mahatma Gandhi Medical College, Pondicherry, India

J Hand Microsurg:2020;12:135-162 


\section{Abstract}

\section{Keywords}

- COVID-19

- consensus

- recommendations

- orthopaedics surgery

- initial and mid-lockdown phases

- rationale treatment
With a lot of uncertainty, unclear, and frequently changing management protocols, COVID-19 has significantly impacted the orthopaedic surgical practice during this pandemic crisis. Surgeons around the world needed closed introspection, contemplation, and prospective consensual recommendations for safe surgical practice and prevention of viral contamination. One hundred orthopaedic surgeons from 50 countries were sent a Google online form with a questionnaire explicating protocols for admission, surgeries, discharge, follow-up, relevant information affecting their surgical practices, difficulties faced, and many more important issues that happened during and after the lockdown. Ten surgeons critically construed and interpreted the data to form rationale guidelines and recommendations. Of the total, hand and microsurgery surgeons (52\%), trauma surgeons (32\%), joint replacement surgeons $(20 \%)$, and arthroscopy surgeons (14\%) actively participated in the survey. Surgeons from national public health care/government college hospitals (44\%) and private/semiprivate practitioners (54\%) were involved in the study. Countries had lockdown started as early as January 3, 2020 with the implementation of partial or complete lifting of lockdown in few countries while writing this article. Surgeons (58\%) did not stop their surgical practice or clinics but preferred only emergency cases during the lockdown. Most of the surgeons (49\%) had three-fourths reduction in their total patients turn-up and the remaining cases were managed by conservative (54\%) methods. There was a 50 to $75 \%$ reduction in the number of surgeries. Surgeons did perform emergency procedures without COVID-19 tests but preferred reverse transcription polymerase chain reaction (RT-PCR; 77\%) and computed tomography (CT) scan chest (12\%) tests for all elective surgical cases. Open fracture and emergency procedures (60\%) and distal radius (55\%) fractures were the most commonly performed surgeries. Surgeons preferred full personal protection equipment kits (69\%) with a respirator (N95/FFP3), but in the case of unavailability, they used surgical masks and normal gowns. Regional/local anesthesia (70\%) remained their choice for surgery to prevent the aerosolized risk of contaminations. Essential surgical follow-up with limited persons and visits was encouraged by $70 \%$ of the surgeons, whereas teleconsultation and telerehabilitation by $30 \%$ of the surgeons. Despite the protective equipment, one-third of the surgeons were afraid of getting infected and $56 \%$ feared of infecting their near and dear ones. Orthopaedic surgeons in private practice did face 50 to $75 \%$ financial loss and have to furlough $25 \%$ staff and $50 \%$ paramedical persons. Orthopaedics meetings were cancelled, and virtual meetings have become the preferred mode of sharing the knowledge and experiences avoiding human contacts. Staying at home, reading, and writing manuscripts became more interesting and an interesting lifestyle change is seen among the surgeons. Unanimously and without any doubt all accepted the fact that COVID-19 pandemic has reached an unprecedented level where personal hygiene, hand washing, social distancing, and safe surgical practices are the viable antidotes, and they have all slowly integrated these practices into their lives. Strict adherence to local authority recommendations and guidelines, uniform and standardized norms for admission, inpatient, and discharge, mandatory RT-PCR tests before surgery and in selective cases with CT scan chest, optimizing and regularizing the surgeries, avoiding and delaying nonemergency surgeries and follow-up protocols, use of teleconsultations cautiously, and working in close association with the World Health Organization and national health care systems will provide a conducive and safe working environment for orthopaedic surgeons and their fraternity and also will prevent the resurgence of COVID-19. 


\section{Introduction}

Since the World Health Organization (WHO) announced the coronavirus (COVID-19) as a dreadful pandemic, there have been many uncertainties and indecisiveness ${ }^{1}$ in the protocols for managing orthopaedic surgery and its allied specialty cases. There has been no uniform standard for the orthopaedic surgical practice because of multifarious differences among countries. Besides, geographical and wide economic variations among countries, adopting different norms to suit their patients, influence of local government or national health care systems and their policies, partial dependence on private health care services, lack of personal protection equipment (PPE) kits, respirators (N95 mask/FFP3), and medicines, and constantly changing protocols during the pandemic crisis influenced the orthopaedics practice to a large extent.

Many countries have perceptibly seen a paradigm shift in their selection of cases and reformulated the operating procedures during this time. Being an emergency or an elective procedure, there is a stringent recommendation among all surgeons across the borders to prevent the risk of coronavirus infection. Overall there is a significant impact of COVID-19 in daily orthopaedics practices, shifting the focus to conservative management. Apart from government hospitals and national health care systems, this reduction in the number of surgeries indirectly had repercussions in the private sectors, producing financial constraints and staff furloughs. For reducing human contacts, surgeons have focused on teleconsultations and telerehabilitation which had considerable consequences on the quality of care and partly abraded moral and ethical responsibility. This article envisages construing the real-time data, facts, patent selection criteria, operating standards, admission and discharge arrangements, safe surgical protocols, difficulties, and the fear faced in social life with inputs from 100 orthopaedic surgeons from 50 countries in the current pandemic situation.

Based on the inputs, we propose rationale and practical recommendations for safe orthopaedics surgical practice. The consensus from these 100 surgeons could pave ways for implementing it as "International Orthopaedic Surgeons' recommendations and consented guidelines for safe surgical practices during this early and mid-phase of COVID-19."

\section{Materials and Methods}

An online survey with a set of questionnaires was sent to 50 countries' volunteered orthopaedic surgeons $(n=100)$ through Google between May 20 and 27, 2020. The responses were kept confidential and interpreted. The participants included were surgeons of various specialties: orthopaedic and trauma, pediatric orthopaedic, spine, arthroscopy, arthroplasty, pelvis and acetabular surgery, hand and microsurgery, shoulder and elbow surgery, and foot and ankle surgery.

The quantitative results obtained from this study were compiled and sent to 10 different orthopaedic surgeons who have 15 or more years of active practice in the field of orthopaedic surgery. The results are qualitatively analyzed and sent back to the corresponding author with several recommendations. Based on both quantitative and qualitative analyses, these recommendations were again proposed to the 100 orthopaedic surgeons involved in the study. A consensus was achieved relying on three types of responses: agreed, disagree, or suggestion/abstain. A 50 to $75 \%$ agree or disagree response formed a majority and strong consensus. Greater than $75 \%$ forms a super majority and a very strong consensus. Besides, valid suggestions and critical corrections were included at this juncture from all participating surgeons to refine the consensuses.

\section{Results}

One hundred orthopaedic surgeons of various subspecialties participated in the study from 50 countries ( - Fig. 1 ). Three surgeons wanted the identity as anonymous. The response was rapid in $45 \%$ of the participating surgeons with a mean of 45 minutes (range: 10 minutes to 23 hours) and remaining responded in a mean 3.5 days. All other surgeons who accepted the survey but could not submit the forms in time due to work schedule and technical issues were excluded from the study. The mean case of the study as on May 27, 2020 was 39,820 (range: $70-336,000$ ). Lockdown was started as early as January 24, 2020 in China, followed by many other countries (-Table 1). Few countries such as Taiwan, South Korea, and Sweden which participated in this study had no official lockdown but followed strict social distancing, avoidance of using public transports and nonessential services, aggressive testing, contact tracing, isolation, and hygienic measures to contain the COVID-19. United Arab Emirates had lockdown between $8 \mathrm{pm}$ and $6 \mathrm{am}$; Turkey had irregular lockdown periods with strict home stay for persons older than 65 years and younger than 20 years ( - Fig. 2 ).

Orthopaedic surgeons working in national health care systems (National Health Service [NHS] trust, etc.) and governmental colleges/hospitals accounted for $44 \%$ of the study. Of which, $50 \%$ were posted in the pneumonia ward. This decision was taken as a government/national health care policy to ameliorate the physician stress and to manage the overloaded asymptomatic but positive COVID-19 patients in the wards. They were also included in the pneumonia wards' rosters to look after the sick patients and monitor the staff nurses and paramedical staffs. They were trained for intubation, but none of the surgeons had any chance to perform or did the study.

\section{Rationale}

Orthopaedic surgeons are at high risk of infection $(1.5-20.7 \%)^{2}$ because of contaminations from colleagues, outpatients' clinics, wards, operating rooms (ORs), and intensive care units (ICUs).

\section{Recommendations}

If they are working in pneumonia wards and other COVID-19 duties, the risk of infections may be exponentially high. Besides, they face the pressure from inadequate protection from contamination, frustration, isolation, exhaustion, and unable to practice the orthopaedic surgery or its allied 


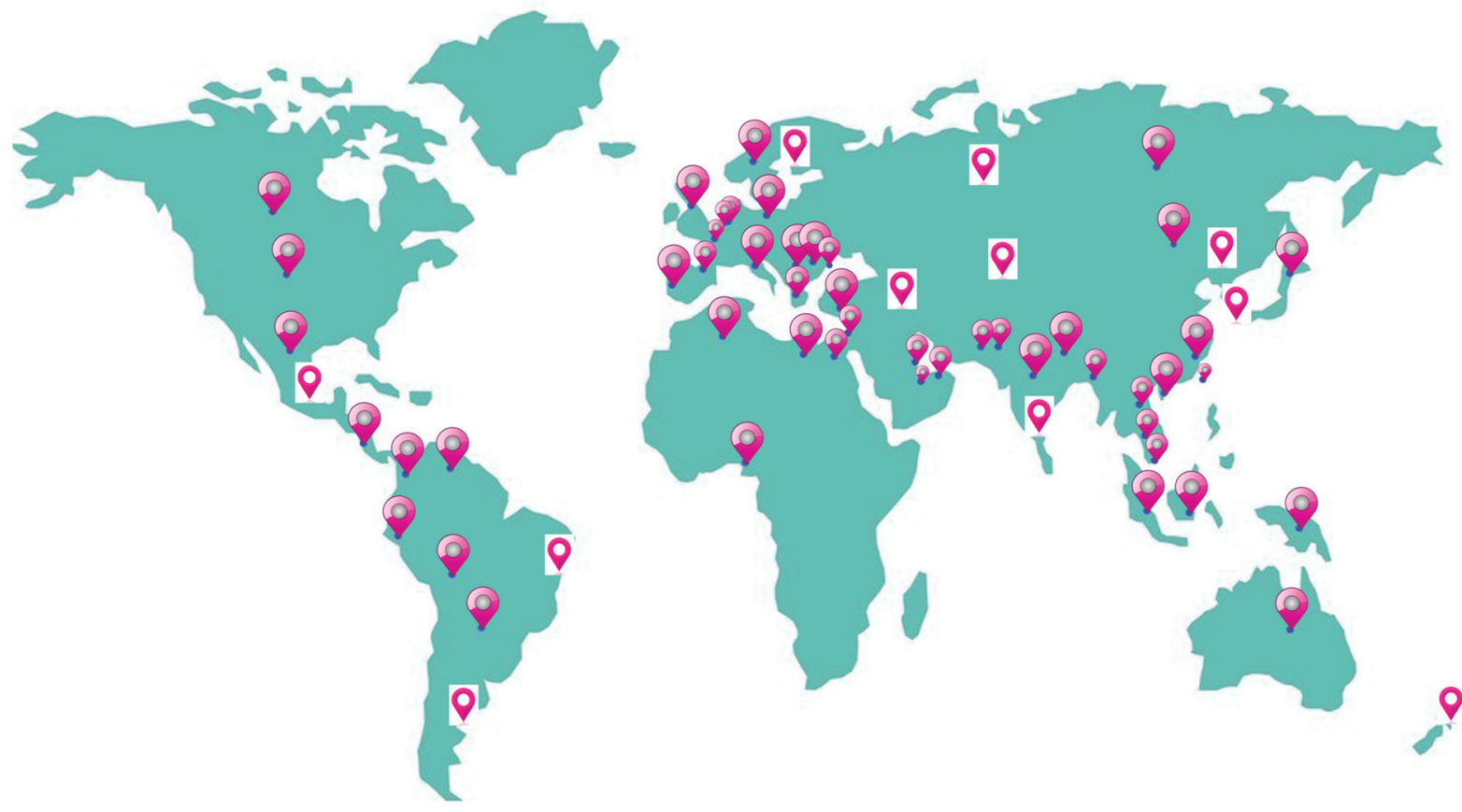

Fig. 1100 orthopaedic surgeons from 50 countries dealing with one COVID-19. A pneumonia of unknown cause detected in Wuhan, China was first reported to the WHO Country Office in China on December 31, 2019. The outbreak was declared a Public Health Emergency of International Concern on January 30, 2020.On February 11, 2020, WHO announced a name for the new coronavirus disease: COVID-19.

Table 1 Current status of lockdown in 50 countries (as of May 31, 2020)

\begin{tabular}{|l|l|l|}
\hline $\begin{array}{l}\text { Complete lift of } \\
\text { lockdown }\end{array}$ & Partial & Phased release/stage manner lockdown countries \\
\hline $\begin{array}{l}\text { Japan, Sweden } \\
\text { South Korea, Taiwan }\end{array}$ & $\begin{array}{l}\text { Argentina, Spain, Egypt, Romania, } \\
\text { Republic of Kosovo, Costa Rica, Croatia, } \\
\text { Germany } \\
\begin{array}{l}\text { Unites States, Portugal, Belgium, Greece, } \\
\text { Iran, Nigeria, Bulgaria, Jordan, Switzerland, } \\
\text { France, Italy, Norway, India, United Kingdom }\end{array}\end{array}$ & $\begin{array}{l}\text { Qatar, Singapore, Malaysia, Colombia, Canada, Indonesia, } \\
\text { Saudi Arabia, Australia, Algeria, Thailand, Nepal, Colombia, } \\
\text { Hong Kong, Pakistan, Libya, Mexico, Bolivia, Poland, Brazil, } \\
\text { Bangladesh, and Peru }\end{array}$ \\
\hline
\end{tabular}

subspecialty. It is prudent and necessary to relocate orthopaedic surgeons and utilize their services at the department concerned.

\section{Level of evidence: $\mathrm{V}$}

Participant vote: $94 \%$ agree; $3 \%$ disagree; $3 \%$ abstain (super majority, strong consensus) (95\% Cl 88 to 97 ).

Private/semiprivate practitioners and orthopaedic surgeons as nursing homeowners accounted for $54 \%$ in the study. They had shut down their practices (27\%) immediate to lockdown and had the option to tailor the clinics and surgeries depending upon the crisis and the need. The missionary hospital (1\%), nongovernment organizations (3\%), and semiprivate hospitals (5\%) were working with poor supply of PPE kits, masks, and other protective accessories. They had worst time in coping up and meeting the daily expenses and working costs. The surgeons working in the private medical college hospitals (21\%) had stopped the nonemergency clinics/cases and continued operating the emergencies and emergency clinics.

\section{Immediate Lockdown Status}

There were many factors involved in the decision making for the orthopaedic surgeons to continue their normal schedule or to stop the clinics or surgeries or both. Countries like United States, United Kingdom (NHS trust), and other public health care systems were operating upon emergent cases, postponing/deferring surgery for nonemergency cases, and consulting selective patients in the clinics (44\%) during the entire lockdown. Of the $54 \%$ private practitioners and self-employed orthopaedic surgeons, $27 \%$ stopped surgery and clinics.

Subsequent to the lockdown, and gradual resumption of work in their country, 35\% surgeons prefer to operate and consult their clinics partly in a phased manner, which consisted of working for 2 weeks and self-quarantining for 2 weeks. Despite some relief and restricted lockdown lift in their countries, private practitioners/nursing homeowners chose the clinics (30\%) and surgery (25\%) to remain shut. A 


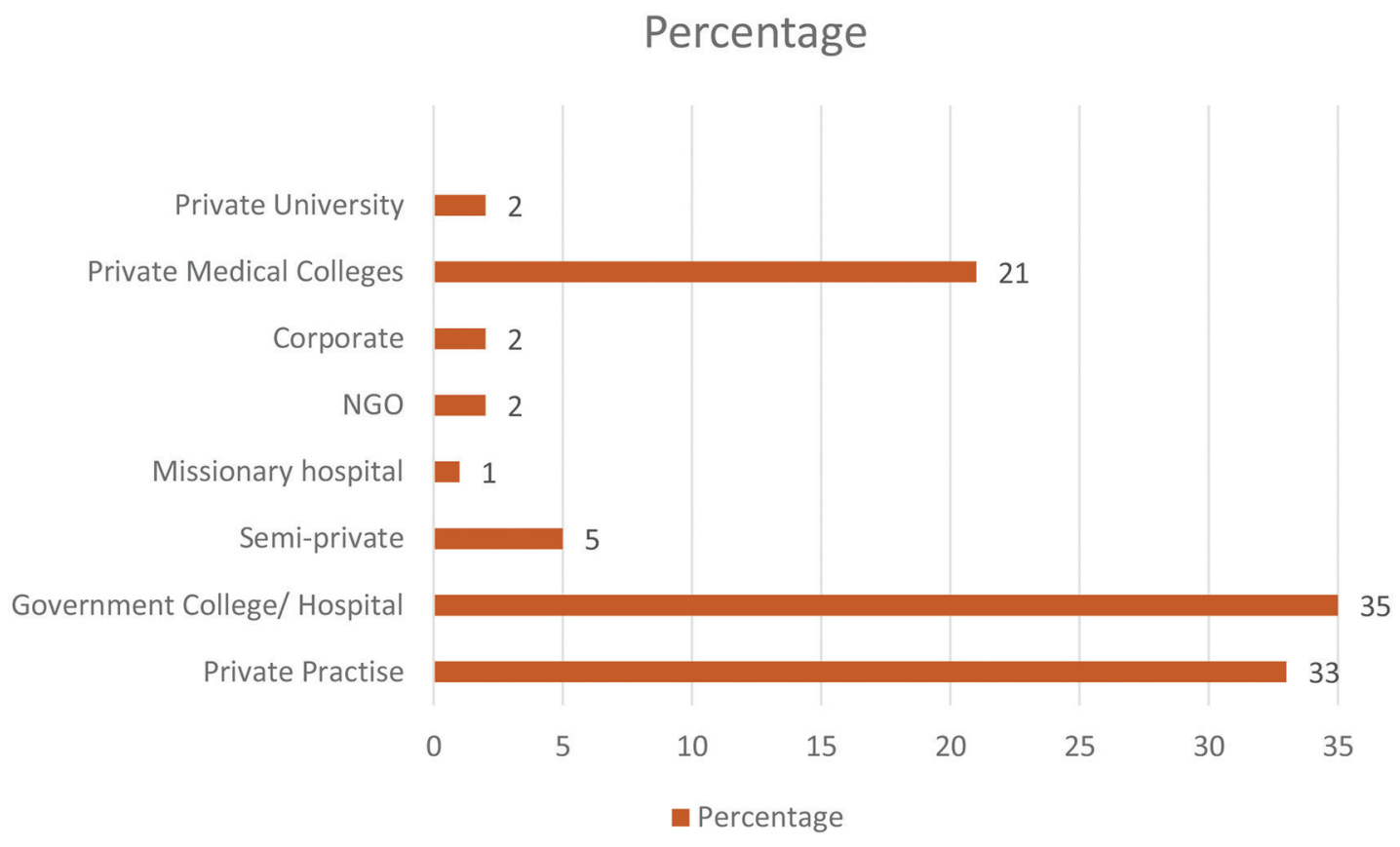

total of $9 \%$ surgeons had completely stopped their orthopaedics practice because of their fear, comorbid conditions, and the age $>60$ years.

More than $55 \%$ of the countries were experiencing some kind of lockdown during the entire study. Japan declared lockdown lift on May 25, 2020 and subsequently many countries such as United Kingdom and India lifted lockdown in a phased/complete manner during the writing of this article. Many countries (54\%) still continue to have lockdown and hope to lift partially or in a staged phase. India had demarcated the infected zones as Green-infections free, Orange-sufficiently infected, and Red-severely affected. This demarcation helped them to lift the ban on services such as local transport, supermarkets and grocery and vegetable supply, and promoted gradual return of life activities: Green zone with free movement within the districts, Orange zone with limited access to food supply, and Red zone remained totally contained and put under strict vigilance. All emergencies were attended in time and referred hassle-free ( - Fig. $\mathbf{3}$ ).

\section{Specialty Surgeons Participated in the Study}

The orthopaedic and subspecialty surgeons involved in the study are compiled in $\boldsymbol{- F i g}$. 4 .

\section{COVID-19 Protocols for Admission}

Surgeons (84\%) working in private/government hospitals had trained paramedical/emergency room (ER) teams which asked for patient's symptoms (fever $>38.5^{\circ} \mathrm{C}$, cough, fatigue, breathlessness, anorexia, malaise/myalgia, loss of taste or smell, sore throat, nasal congestion, headache, diarrhea, nausea, vomiting), travel history, contact history, locality (containment zones) in the hospital reception. If found negative they were registered and allowed to enter inside the hospital premises. Suspected cases, symptomatic patients (44\%), and positive travel history patients were isolated, separated, and referred to fever clinic/tertiary government medical college hospitals for COVID-19 tests and further management. Orthopaedics teams (73\%) monitored temperature, oxygen saturation, pulse rates, and blood pressure before sending them to clinical consultation rooms or ERs. Strict adherence of 2 meter distancing, frequent hand wash with $70 \%$ alcohol-based sanitizers, and no visitor for ambulant/single visitor for nonambulant patients (53-60\%) were mandatorily followed in 100\% of surgeons' working places. As a precautionary measure, $44 \%$ of patients were contacted through telephone and enquired about the symptoms and travel history. If suspicious symptoms elicited, they were counseled for postponing surgery and treat them conservatively with medicines 


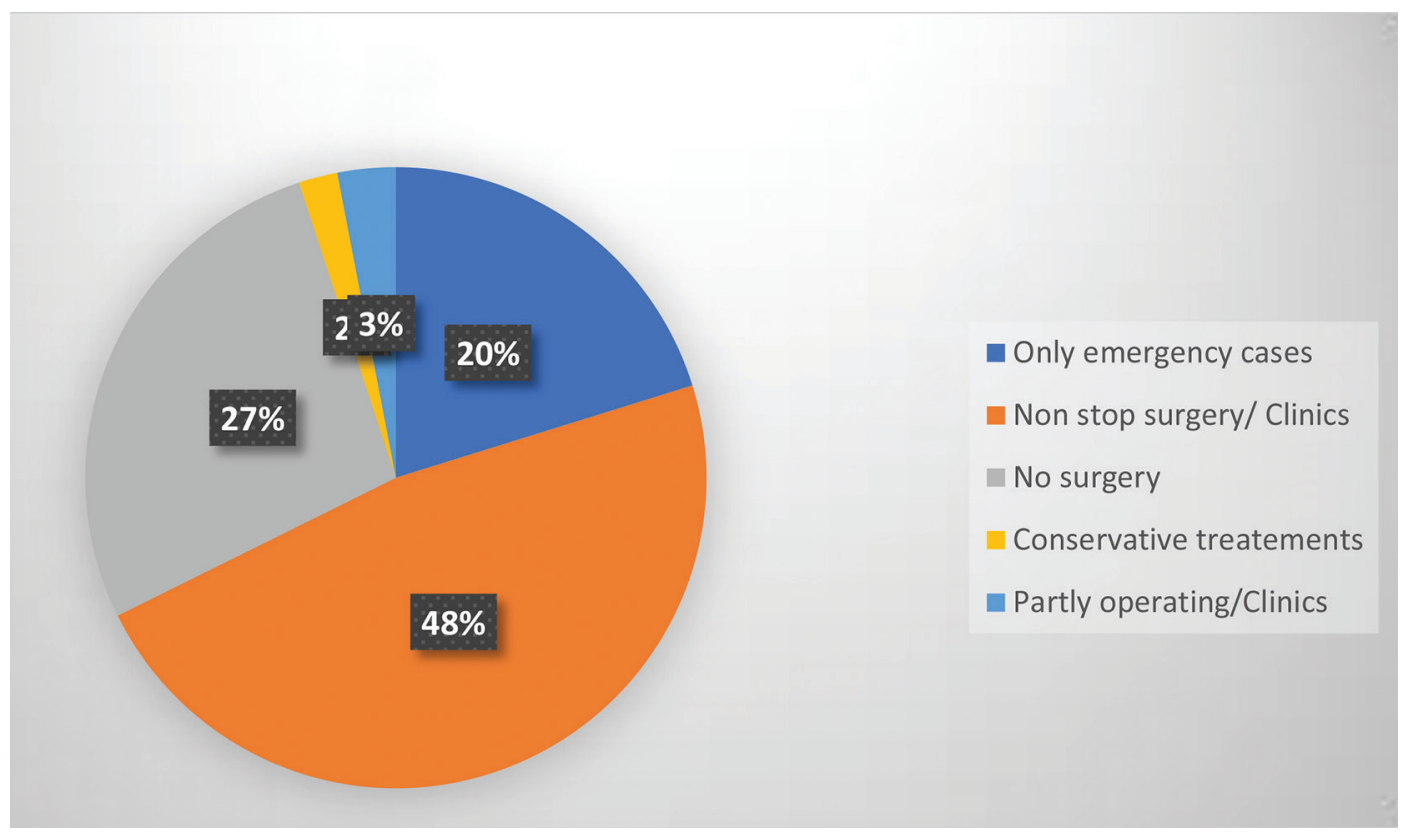

Fig. 3 Lockdown status among orthopaedic surgeons.

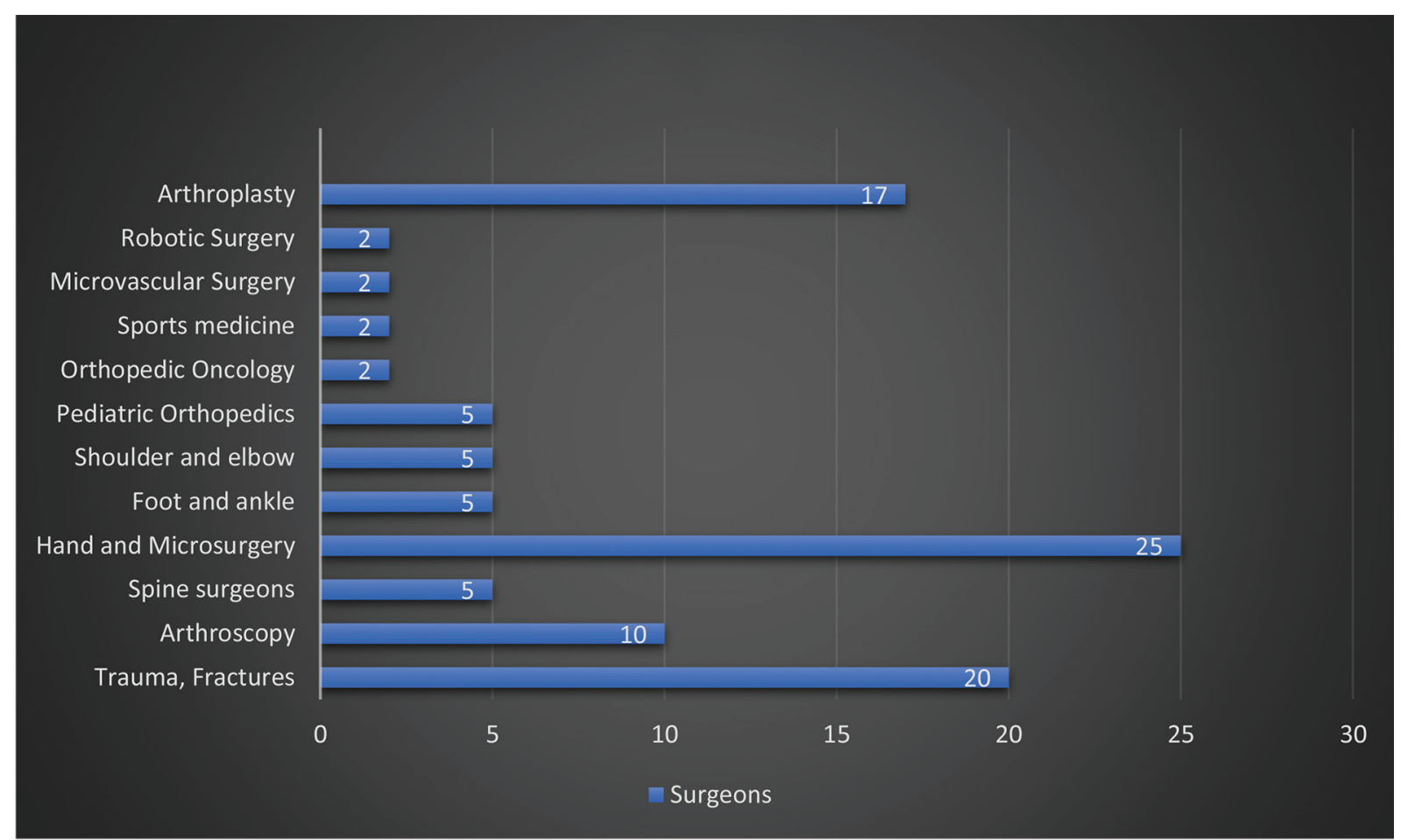

Fig. 4 Orthopaedic and subspecialty surgeons involved in the study. 
and splints (22\%). In total, $44 \%$ surgeons preferred day care surgery. A total of $42 \%$ of hospitals had separate COVID-19 rooms for admitting suspected COVID-19 cases (40\%) and protocols for checking bed availability at any given point in time (-Fig. 5).

\section{Rationale}

WHO has published a recommendation for health care workers based on the current knowledge of the situation in China and other countries. ${ }^{3}$ It has laid strategies to prevent or limit transmission. WHO and Center for Disease Control and Prevention (CDC) have both laid guidelines for prehospital survey $^{3}$ and work-up. ${ }^{4-6}$

\section{Recommendations for Preoperative Work-Up}

COVID-19 risk profile, travel history, and contact history should be collected and scrutinized. Temperature and oxygen saturation should be checked on the day of surgery (-Fig. 6). If positive findings (fever $>38.5^{\circ} \mathrm{C}, \mathrm{SpO}_{2<90} \%$ on room air) are found, the patients should be evaluated with diagnostic work-up before surgery (reverse transcription polymerase chain reaction [RT-PCR] and computed tomography [CT] scan chest).

Level of evidence: $\mathrm{V}$

Participants vote: agree: 94\%, disagree: $3 \%$, should be done before surgery: 3\% (super majority, strong consensus) (95\% CI 88 to 97 ).

\section{Management of Nonemergency Cases}

Considering the national emergency and critical lockdown, 55\% of surgeons deferred surgery and adopted alternative/conservative methods of treatment. Local steroid injections, splints, cast, and oral analgesics were given to patients during the pandemic. Among the nonemergency cases, 37\% surgeons found that they had one or more symptomatic patients who were referred to government/ private tertiary medical colleges for further evaluation and management. Only 3\% surgeons operated on nonemergency cases such as radial tunnel syndrome where working women presented with severe pain restricting their daily activities. Immediate to the surgery, they returned to normal work and were pain-free and happy (-Fig. 7).

\section{Rationale}

The Centre for Medicare and Medicaid Services (CMS) has given preferential recommendation for surgery and conservative management. ${ }^{7}$ We had modified the questionnaire to suit the orthopaedics practices. They are as follows:

- Low acuity treatment: carpal tunnel syndrome, trigger finger, tennis elbow, etc.

- Intermediate: joint replacement, spine surgery, arthroscopy.

- High acuity: open fractures, severe trauma-fractures and dislocations, cauda equina syndrome, compartment

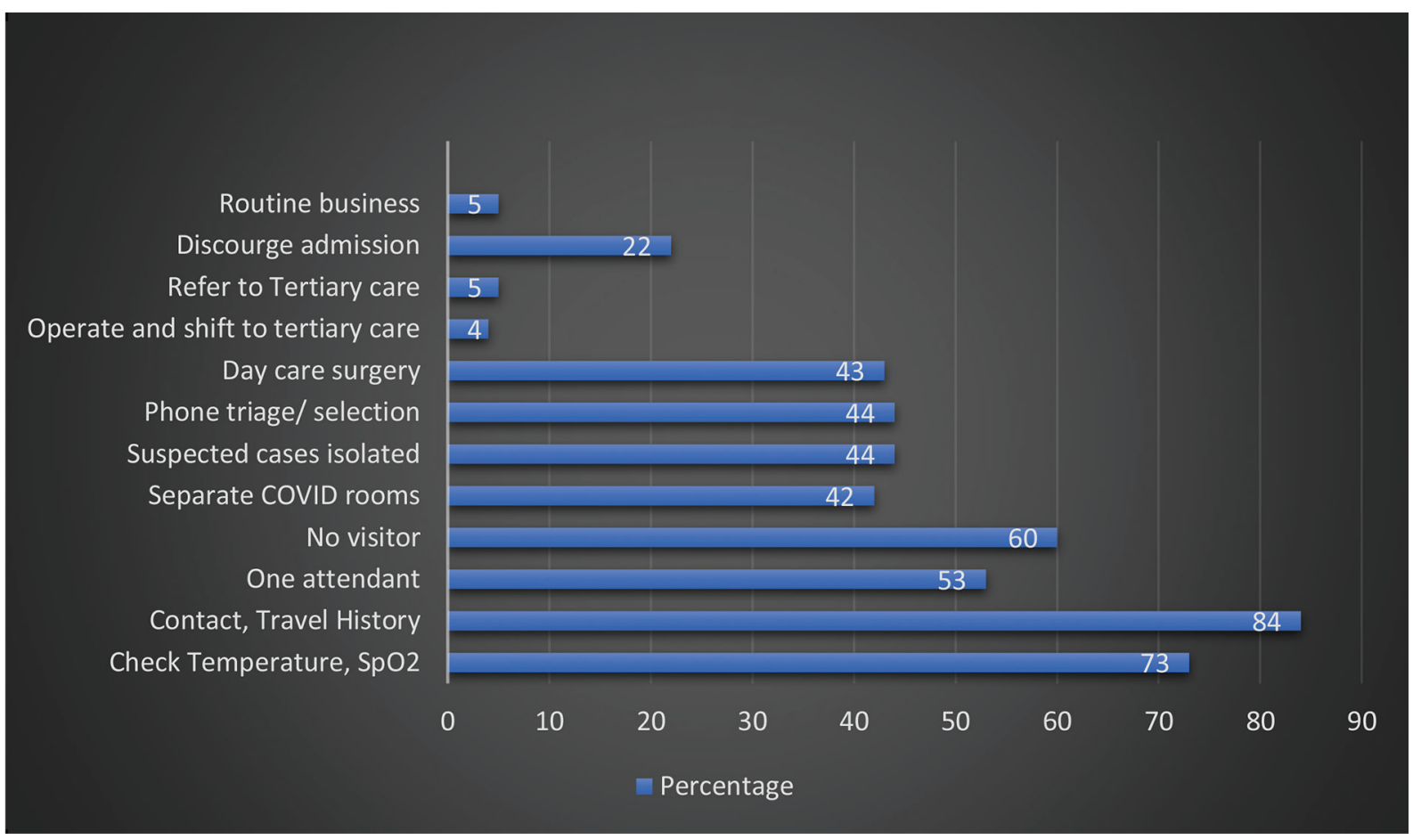

Fig. 5 COVID-19 protocols for admission. 


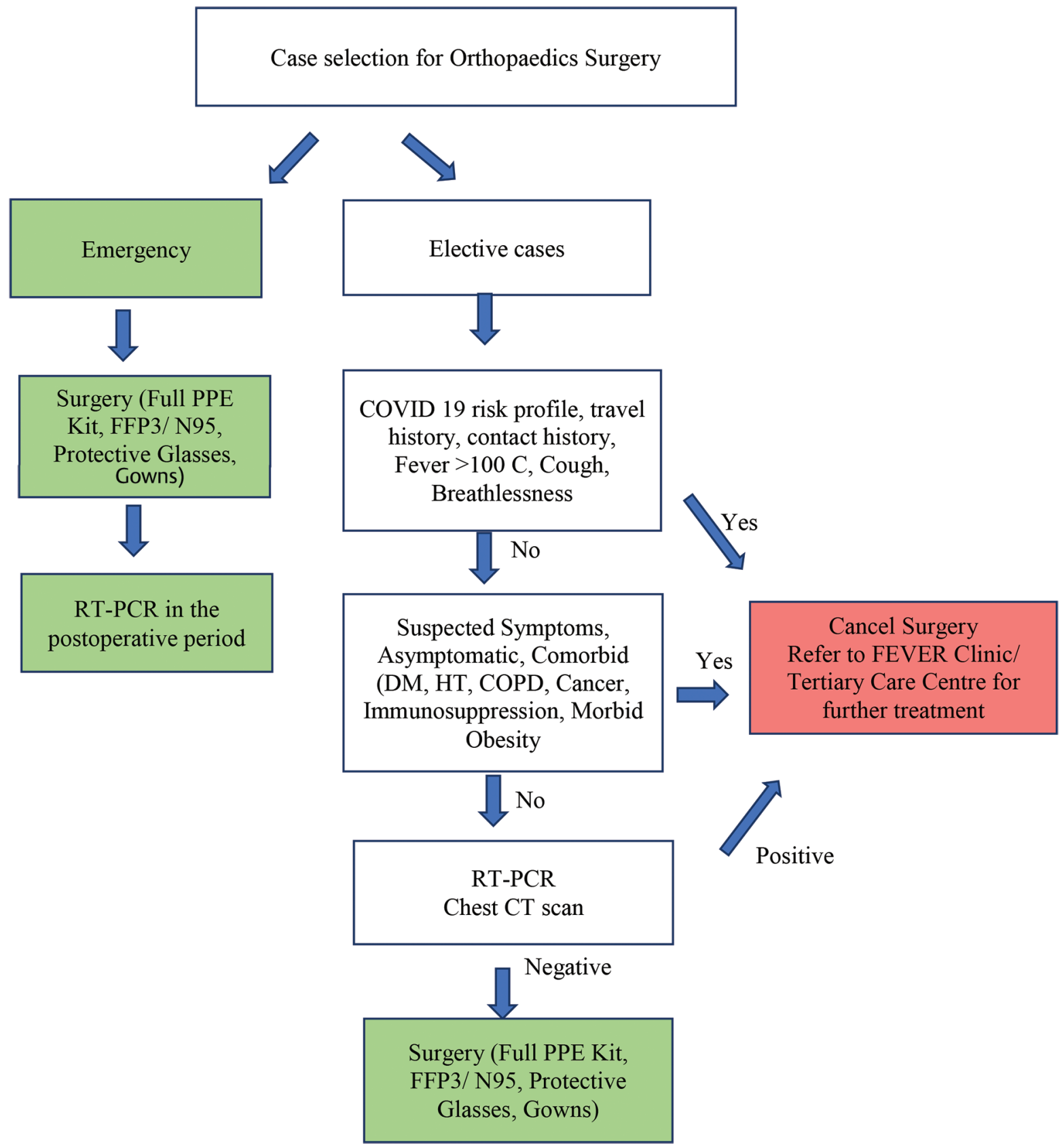

Fig. 6 Recommendations for preoperative work-up (flow chart).

syndrome, cancer, highly symptomatic, acute infections, necrotizing fasciitis, and vascular injuries (-Fig. 8).

\section{Recommendations from Surgeons}

- Low acuity: conservative/steroid injections/oral analgesics/ splints.

- Intermediate: surgeons' discretion.

- High acuity: surgery.

Level of evidence: $\mathrm{V}$

Participants vote: $97 \%$ agree, $3 \%$ disagree (super majority, strong consensus) (95\% CI 92 to 99).

\section{Patients' Consent and Information}

Patients received information about COVID-19, hand hygiene, and the safe hygienic practices prior to surgery. In addition, surgeons also obtained prior informed consent (87\%) and special consent (49\%) in bilingual version (English + native language) about the risk of contamination and spread to them during the stay. As a mandatory practice, patients wore surgical mask and protective kits during the stay in the hospital. All elective patients received adequate counseling before surgery (66\%) and chances for postponement and counseling for nonemergency case delaying were rendered. 


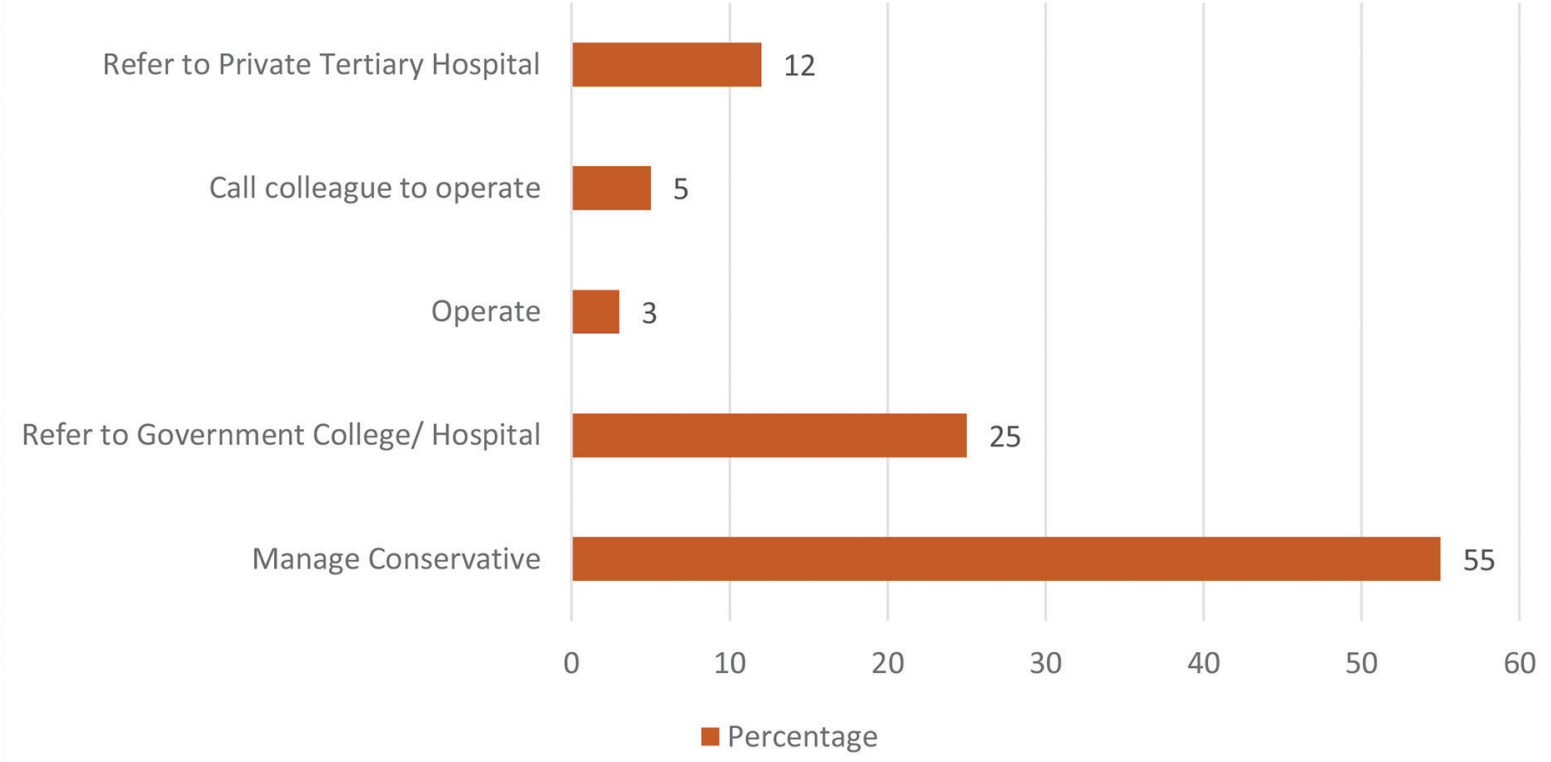

Fig. 7 Management of nonemergency cases.

\section{Surgeries Performed during the Lockdown}

Distal radius fractures (53\%), hand and carpal bone fractures/dislocations (57\%), forearm fractures (39\%), elbow fractures/dislocations (40\%), shoulder (24\%), spine fractures, paraplegia and dislocations (17\%), pelvis and acetabulum (15\%), hip fractures/dislocations (46\%), femur fractures (32\%), knee (27\%), leg, ankle, and foot fractures (28\%), microsurgeries (29\%), fingertip injuries (45\%), soft tissue injuries (37\%), amputations (39\%), replants and revascularizations (30\%), septic arthritis/infections (38\%), tendon, nerve, and muscle injuries (50\%), open fractures (57\%), and emergency surgeries (57\%) happened during the COVID-19 crisis in 50 countries. In total, 75\% surgeons did all these surgeries in their normal OR; 15\% had COVID-19 makeshift ORs where the cases were done; $<10 \%$ had the surgery done in minor ORs or day-care units or adjacent to ORs ( - Fig. 9).

\section{Orthopaedic Surgery for Elderly and Comorbid Conditions}

Orthopaedic surgeons had many challenging tasks in operating on elderly patients invariably associated with comorbid conditions (diabetes, hypertension, coronary artery disease, cancer, immunosuppression, and moribund obesity). Domestic falls at home were the main reason for fractures in the elderly population. Distal radius fractures (20\%), trochanteric fracture (35\%), fractured neck of femur (25\%), supracondylar femur fractures (12\%), surgical neck humerus fractures (10\%), ankle fractures/ dislocations (7\%), lumbar fractures (5\%), acetabular fractures (3\%), and other fractures involving the hand and foot were treated by the surgeons involved in the study. Hip fractures and hip and knee surgeries in elderly patients had $15 \%$ mortality in this study because of worsened varied existing reasons such as chronic renal failure, urosepsis, and coronary artery diseases. Most of the surgeons (90\%) treated distal radius fractures, surgical neck humerus fractures, and hand fractures conservatively with cast/splints and early mobilization.

Both in adults and elderly patients, these 100 surgeons had followed certain protocols before and after the surgery. Based on their suggestions, we propose directions for the presurgical work-up including for emergency and elective cases and the rationale behind.

\section{Rationale}

Due to their increased susceptibility to pulmonary infection and increased morbidity ${ }^{8,9}$ because of being bed-ridden and associated comorbid conditions such as hypertension, heart disease, or diabetes, ${ }^{10,11}$ orthopaedic surgeons operate on the elderly population to enhance early mobilization and prevent risk of deep vein thrombosis (DVT), ${ }^{12}$ thereby reducing the morbidity and mortality. Given the COVID-19 scenario, elderly patients are vulnerable to developing pneumonia after fractures. So high suspicion with a definite treatment guideline for elderly patients is required. ${ }^{13,14}$ Open fractures and severe injuries require emergency surgery. ${ }^{15,16}$

\section{Recommendations}

- Conservative for distal radius, surgical neck humerus, and clavicle fractures. 


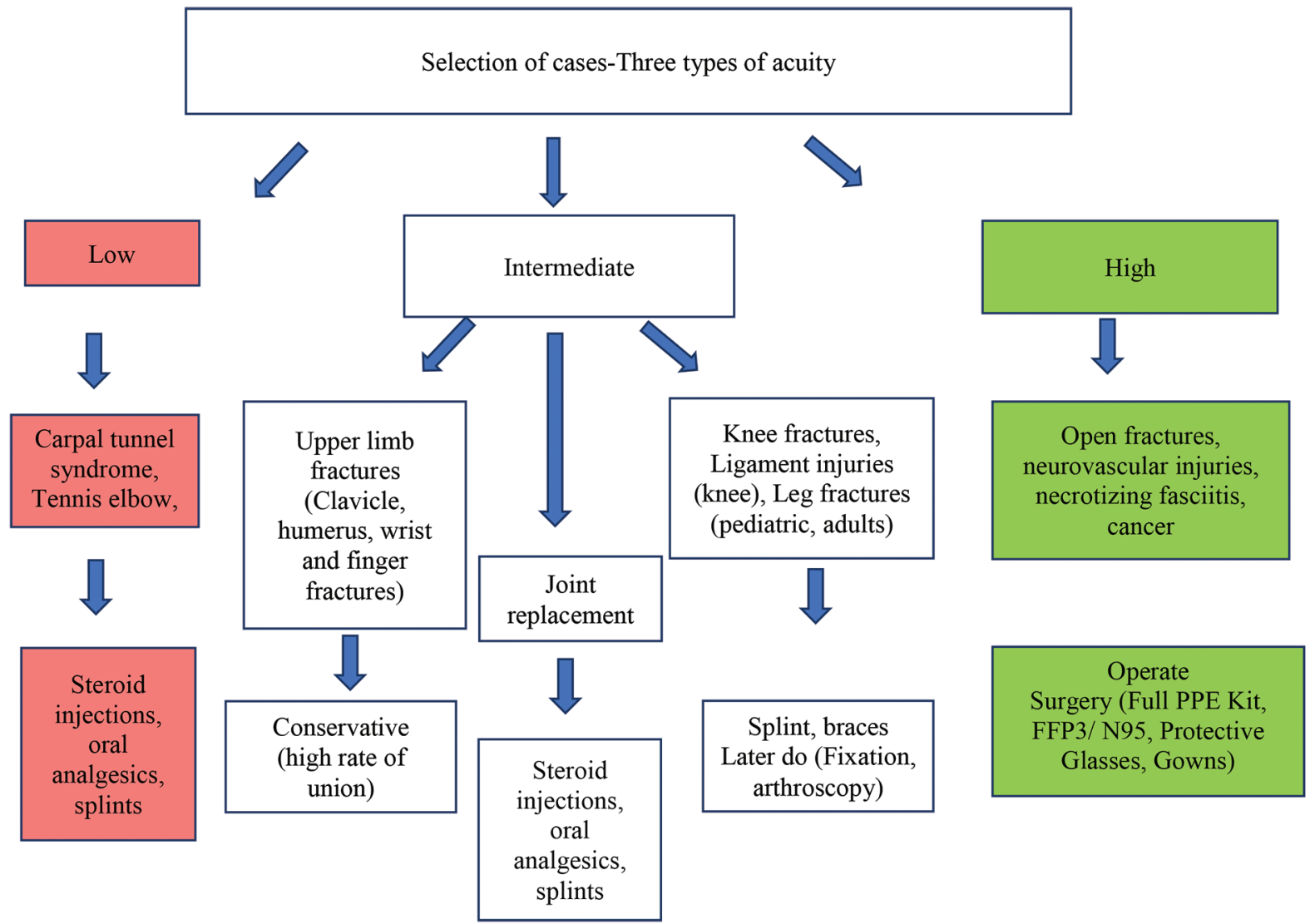

Fig. 8 Suggested flow-chart recommendation for selection and management of cases.

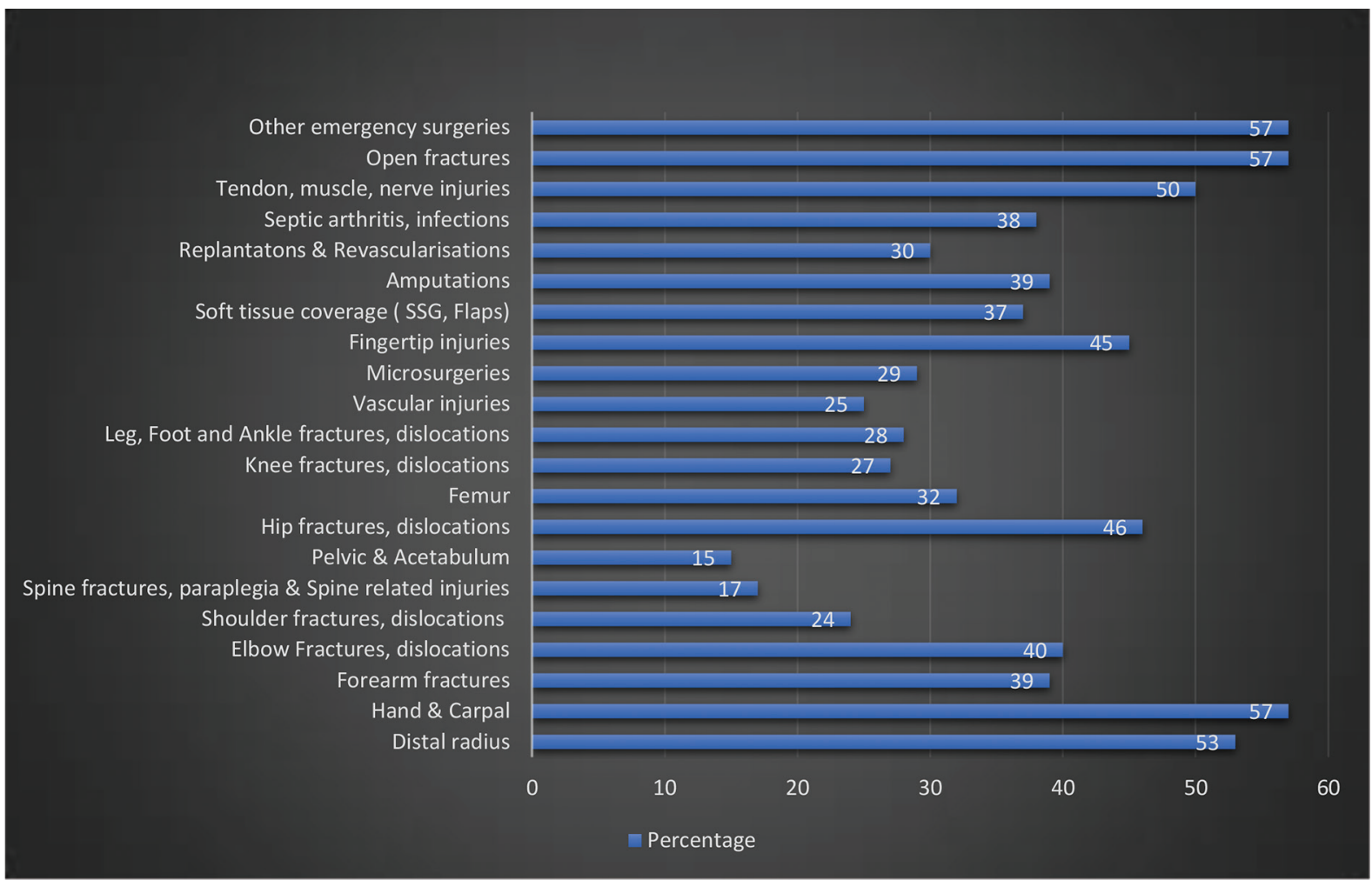

Fig. 9 List of surgeries done (total: 637 cases). 
- Operative for hip and knee fractures to have early mobilization and prevent morbidity and mortality, DVT, and pulmonary complications.

- Strict infection control measures, anticoagulation, and intensive supportive care.

Level of evidence: $\mathrm{V}$

Participants vote: agree: 78\%, disagree: 13\%, abstain: $9 \%$ (super majority, strong consensus) [95\% CI 69 to 85].

\section{Orthopaedics Cases and Percentage of Reduction during the Lockdown}

When analyzing the number of surgeries in different countries during the lockdown, marked reductions were noted uniformly. Surgeons (18\%) performed less than five surgical cases per month; $<5-15$ cases in 24\%; $16-25$ cases in $22 \%$; $26-50$ cases in $18 \% ; 51-100$ cases in $8 \%$, and $>100$ cases in $8 \%$. Ten percent of surgeons did not operate during the entire lockdown period. More than half of the surgeons had 50 to $75 \%$ reduction in patients' turn-up for regular clinics.

Again, countries with public health care systems modeled with the government, general taxations, ministry of health control, or insurances, such as United States, United Kingdom, United Arab Emirates, Saudi Arabia, South Korea, Taiwan, and New Zealand had been continuing to operate more than 100 cases per month. Countries with huge populations such as China, India, and Pakistan which depend on partly private and government support had marked reduction in the number of cases (5-25 cases per month). Overall, 63\% of surgeons felt three-fourths reduction in their surgical cases compared with their routine schedule ( $\mathbf{- F i g . ~ 1 0}$ ).

\section{Orthopaedics Case Postponement and Catch-Up Procedures}

The surgical case reduction automated to backlogs increases their future surgical loads. Worryingly, surgeons expected $27 \%$ ( $n=25$ ) of patients will not come back for surgery again because of partial relief from conservative management or very long reappointment schedule. On the other hand, if the backlogs kept increasing over a period of time, the surgeons feared burden on the health care systems and catch-up procedures could be hectic and clogged. In total, 50 to $75 \%$ of surgeons estimated catch-up procedures could happen in 3 to 6 months or even longer. They firmly believed that mandatory structural reorganization in the entire health care system to deal backlogs of waiting patients must be done diligently and prudently.

\section{Rationale}

There have been various discussions in the past on how to address backlogs of surgical cases, whether to increase or extend the operating time into the weekends. ${ }^{17}$ The immediate concern from the operating team is physical and mental fatigue because of sudden increase in work burden. Because of this fatigue there is $22 \%$ increased risk of medical error. ${ }^{18}$ Janhofer et al ${ }^{18}$ came up with the solution of intraoperative "microbreaks" for fatigue mitigations and enhanced safety. Besides the authors strategized,

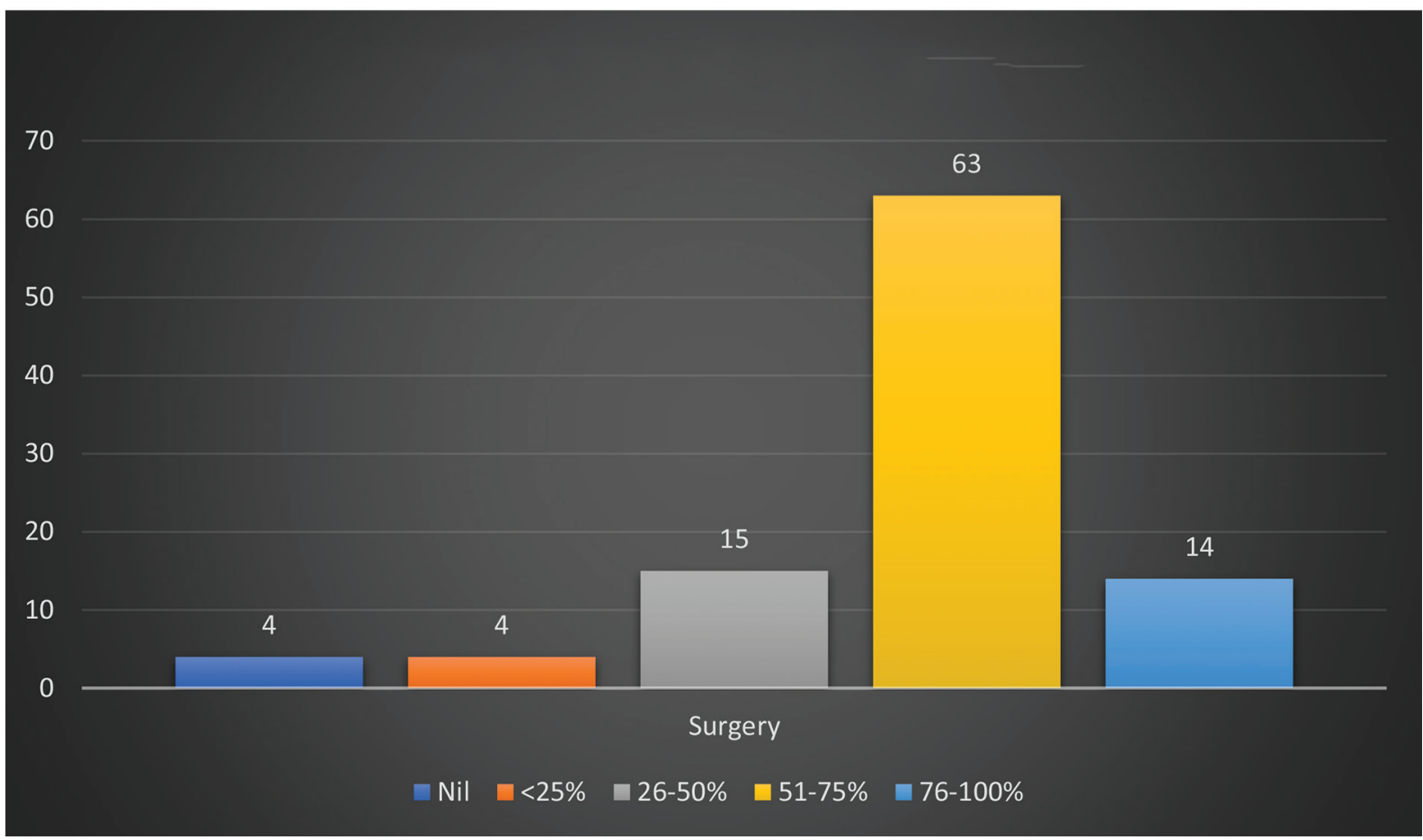

Fig. 10 Surgery reduction during the lockdown. 
surgeons have reduced responsibility, optimized sleep, nutrition, and hydration to maximize surgeon endurance. ${ }^{19}$

\section{Recommendations for Catch-Up Procedures in Orthopaedic Surgery}

- Semi-elective cases and intermediate/high acuity should be given preference for surgical work-up in the catch-up surgical list.

- Considerations should be given to surgeons and the team for mitigating physical and mental fatigues.

- A transparent model should be followed.

\section{Level of evidence: $\mathrm{V}$}

Participants vote: agree: $97 \%$, disagree: $3 \%$ (super majority, strong consensus) (95\% Cl 92 to 99) ( - Fig. 11).

\section{Operating Room Dress Code}

The orthopaedic surgeries were performed in their normal ORs in 73\% and COVID-19 makeshift ORs with negative pressure control and filters in 18\%. Minor ORs and emergency ORs were used in $8 \%$. More than one-third of the surgeons (40\%) did surgery with full PPE kits, N95 masks, face shields, shoe covers, and protective glass, with proper donning and doffing techniques before and after procedures. Surgeons (25\%) performed surgeries with normal surgical masks, operating gowns, shoes, and usual accessories during and after the procedures, partly attributing to nonavailability, poor supply, and increased demand for the PPE kits and the accessories. More cautiously 5\% wore both surgical and N95 masks together during the procedures. Surgeons had perspirations, heat, fogs, and occasional breathlessness wearing the PPE kits and the accessories. Surgeons (2\%) preferred (filtering face piece level 1, 2, and 3) FFP3 masks over N95 masks, which are slightly better and advantageous than N95 masks.

\section{Rationale}

PPE kits include surgical gloves, water-resistant gowns with long sleeves, a surgical mask, and full-face protection with a face shield. This reduces intraoperative wound contaminations from blood and body fluids, which get sprayed in an area of 2 to 8 meters around the operating table. ${ }^{20}$ There are four levels of safety in gowns: level 1 (use in minimal risk environment), level 2 (low risk procedures), level 3 (moderate risk), and level 4 (high-risk procedures/infectious diseases). There are three types of face masks protecting the mouth and the nose.

- Single-use face mask: it filters large particles of $3 \mu \mathrm{m}$, prevents droplet transfer, and is used by the health care workers $^{21}$ to protect and patients to limit COVID-19 transmission. $^{22}$

- Respirators mask ${ }^{23}$ : it filters small particles of 0.3 $\mu \mathrm{m}$ and protects against airborne transmission. The European Standard (EN 149:2001) classifies respirator masks into three types: FFP1, FFP2, and FFP3. The N95 mask is FFP2 type which has 95\% filtering capacity and

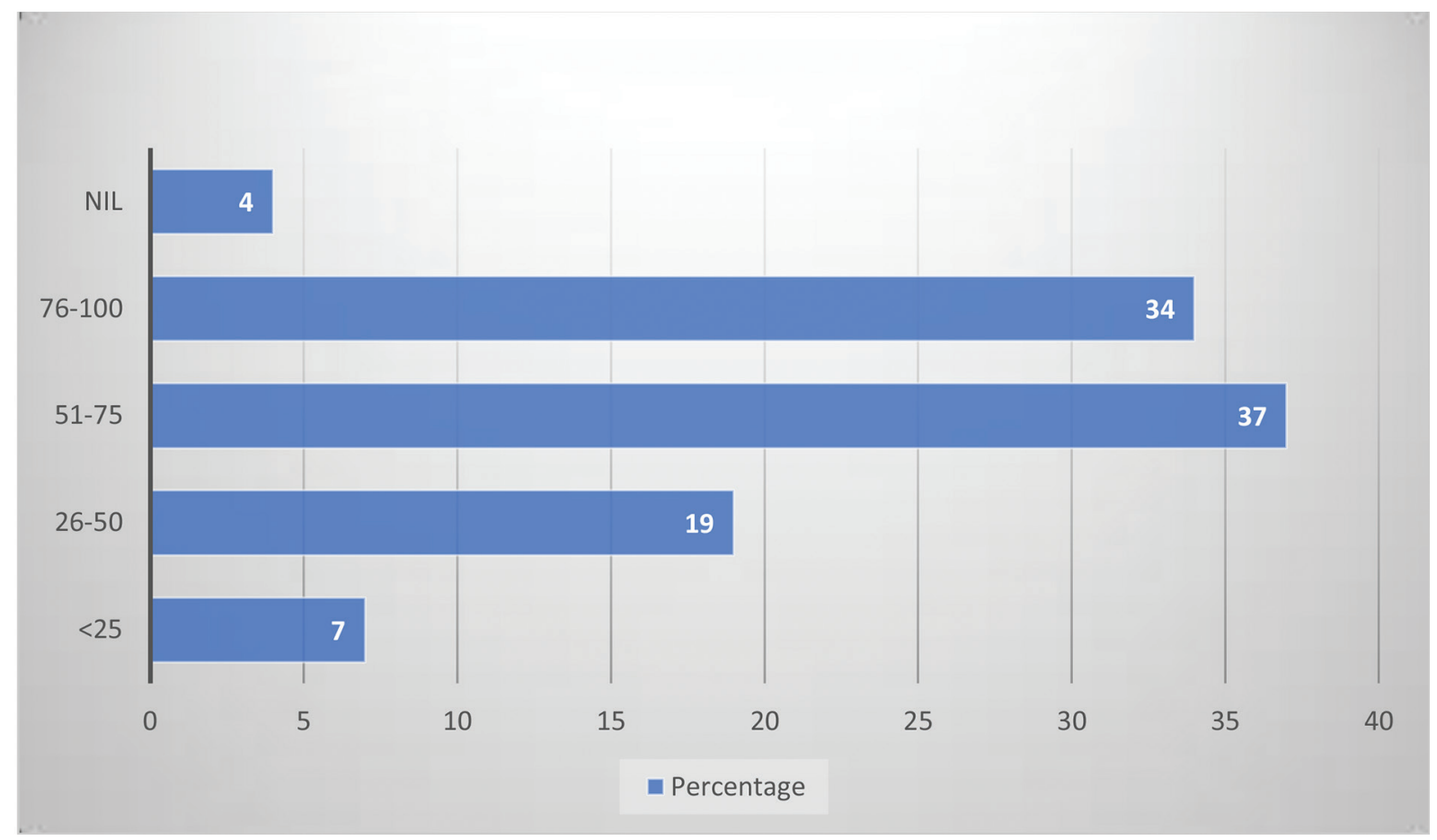

Fig. 11 Postponement of orthopaedic surgery. 
provides good protection. FFP3 is an N99 mask, which gives $99 \%$ filtration against airborne contamination of $0.3 \mu \mathrm{m}$ particles.

- Power air-purifying respirator ${ }^{24}$ : it was used mainly during the severe acute respiratory syndrome (SARS) outbreaks by the health care workers and persons with high risk of transmission (surgeons).

\section{Recommendations}

- Respirators (FFP1, FFP2, and FFP3) are designed to protect against droplets and aerosols (percentage of filtered particles $\geq 300 \mathrm{~nm}$ ). N95 masks filter $95 \%$ of $\geq 300 \mathrm{~nm}$ particles. Both have high protective potential. WHO recommends all heath care workers should wear a respirator (FFP3/N95).

- Surgical masks are reasonably safe for patients with COVID-19 and health care providers.

\section{Level of evidence: $\mathrm{V}$}

Participants vote: $76 \%$ agree, $20 \%$ disagree, $4 \%$ abstain (super majority, strong consensus) (95\% CI 69 to 86).

\section{Operating Team}

Surgeons, OR staff, technicians, radiograph and anesthetist technicians, assistants, ICU staff, and paramedical staff play a crucial role in a successful surgery. Operating orthopaedic surgeon, staff, technicians, and ICU staff worked on roster (28\%) and were quarantined for 14 days. In countries (45\%) with huge cases of orthopaedics trauma cases, COVID-19 cases, and lack of adequate doctor/staff ratios, the entire team worked on regular schedule without quarantine or break. The operating team worked with PPE kits, N95 masks, and protective accessories (25\%) and with normal surgical masks and gowns (45\%).

\section{Rationale}

The Systems Engineering Initiative for Patient Safety (SEIPS) model ${ }^{25}$ provides a good framework for a health care system to critically evaluate the armamentarium of measures to minimize the risk of intrahospital spread and protect its frontline health care workers against occupational COVID-19 infection. It suggested health care workers including doctors to be segregated into two dimensions and the need to get quarantine themselves at regular intervals. ${ }^{26}$

\section{Recommendations}

Doctors, staff, and paramedical staff should be divided into teams working on duty rosters with full PPE kits and N95 masks or equivalent. Medical students should be withdrawn from surgical postings. Daily temperature and $\mathrm{SpO}_{2}$ check should be done. Self-quarantine for 2 weeks is mandated. If symptomatic they need to visit fever clinics for further management.

Level of evidence: $\mathrm{V}$.

Participants vote: $86 \%$ agree, $11 \%$ disagree, $3 \%$ partly (super majority, strong consensus) (95\% CI 78 to 91 ).

\section{Operating Room}

Majority of the surgeons (80\%) recommended limited staff and associates inside the OR. They preferred negative pressure ventilation (32\%), high-efficiency particulate air (HEPA) filters (41\%), and centralized (32\%) and split air-conditioning (16\%). They also insisted for single-use disposable gowns, masks, and accessories (38\%), and strict adherence to autoclaving and implant handling (32\%). Poor supply of disposable gowns and PPE kits, shortage of implant supply, obligatory use of HEPA filters with frequent air changes, and unenduring change of OR infrastructure such as centralized air-conditioning and positive pressure ventilation to negative pressure ventilation were some of the challenging tasks of the surgeons involved in this study to render safe orthopedics surgical practice during this COVID-19 pandemic (-Fig. 12).

\section{Rationale}

ORs are designed with positive pressure and changing it to negative pressure ORs is not easy and cumbersome. ${ }^{25,27}$ Addition of HEPA filters will filter coronavirus $(0.125 \mu \mathrm{m})$ efficiently and reduces viral load and risk of viral disseminations. ORs have air-conditioning and humidification units with individual atmospheric air inlet and exhaust systems. In positive pressure ventilation, fresh air is supplied to the OR without recycling, and the outflow to the atmosphere occurs via overhead exhaust vents. Improvisation of the existing ORs to produce negative pressure ventilation can help reduce viral dissemination. ${ }^{28}$ Nonessential materials are kept away and the operative personal should be reduced to minimum. The rationale behind strict adherence was to prevent the risk of contamination and spread of corona virus. ${ }^{28,29}$ High-speed cutters in cervical spine surgeries and hip replacement surgeries produce aerosol $<5 \mu \mathrm{m}$ and contaminate OR personals. ${ }^{30}$ The blood aerosols can reach the upper respiratory tract. ${ }^{31}$ Pulsatile lavage will splatter blood and body fluids which are highly infectious. ${ }^{32}$ Electrocautery used in surgery which produces smokes (aerosols) ${ }^{16}$ is found to have Corynebacterium, papillomavirus, human immunodeficiency virus, and hepatitis B..$^{33}$ Although limited data are available regarding the corona virus spread while performing such aerosol-generating procedures (AGPs) in orthopaedic surgeries, they should not be any exception.

The indication for surgical fixation during this pandemic should be simple, rational, definitive, and expeditious. ${ }^{34}$ Joint replacement allows early mobilization. Minimally invasive external fixation should be tried for closed and open fractures. ${ }^{35}$ Uncemented ${ }^{36}$ and unreamed nails have shorter operative time and potential advantage in reducing respiratory complications and fat embolism. ${ }^{37}$

\section{Recommendations}

- HEPA filter, dedicating space for nonessential materials inside the OR complex, separate entry and exit rooms with hand washing, stern donning and doffing methods, meticulous and judicious use of high-speed drills, electrocautery, suction devices, definite surgical procedures (early mobilization), less aggressive external fixators for complex injuries, implant choices (uncemented implants, 


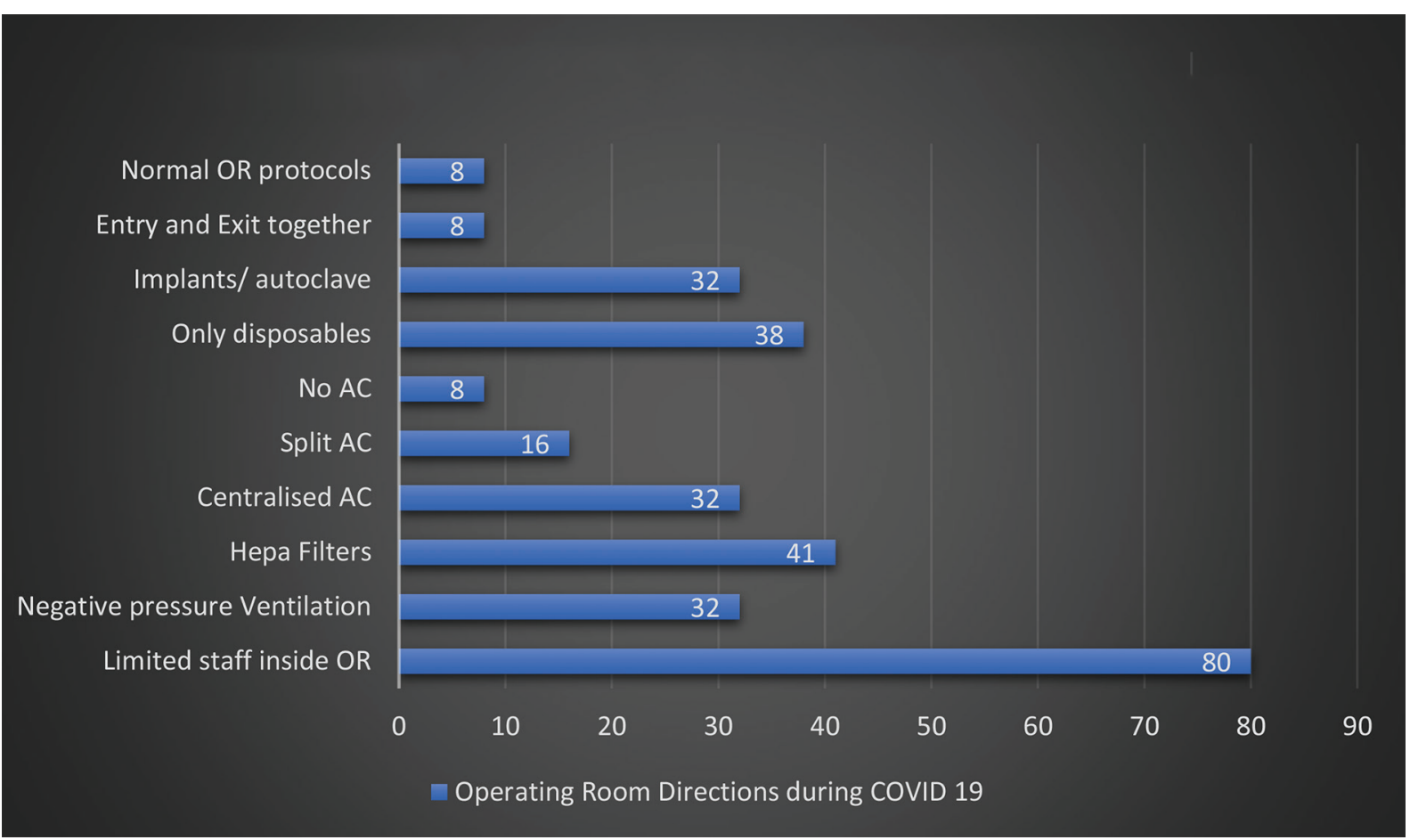

Fig. 12 Operating room recommendations.

unreamed nails), and wound wash techniques (pulsatile lavage) are imperative protocols in the OR complex.

- Cleansing should be done using detergent and water followed by use of 1,000 ppm bleach solution for all hard surfaces in the operating theater. The disinfection time should be longer than 30 minutes. The operating theater should be closed for at least 2 hours, and the next operation should be performed after laminar flow and ventilation being turned on.

- The surgeon should remove the gowns, discard used PPE, adhere to hand hygiene, and shower before leaving the OR complex.

- If possible reverse engineer for negative pressure ventilation ORs.

\section{Level of evidence: $\mathrm{V}$}

Participants vote: agree: $91 \%$, disagree: $6 \%$, abstain: $3 \%$ (super majority, strong consensus) (95\% Cl 84 to 95).

\section{Anesthesia}

Surgeons (60\%) preferred regional anesthesia (spinal/epidural/supraclavicular block, axillary block) over general anesthesia for lower limb and upper limb cases with minimal or no sedation. Surgeons (25\%) did surgeries of the fingertip injuries and finger fractures under local anesthesia and preferred WALANT anesthesia for hand and wrist injuries (tendon repairs/transfers). Surgeons (15\%) opted for general anesthesia when operating head injuries, spine injuries, polytrauma multiple fractures with special precautions during and after intubation. Given that COVID-19 appears to be transmitted via aerosolized droplets with closest person to person contact, all surgeons felt universal precautions and droplet cautions must be followed. The choice of anesthesia technique generally depends on the procedure and patient. All the patients were given surgical masks during the procedure. Surgeons avoided general anesthesia, airway instrumentation, and attendant aerosol generation to prevent risk of airborne transmission (-Fig. 13).

\section{Rationale}

The major routes of SARS-CoV-2 transmission are through respiratory droplets and contact with contaminated surfaces. ${ }^{38}$ The AGPs are tracheal intubation, noninvasive ventilation, tracheostomy, manual ventilation before intubation, and bronchoscopy. ${ }^{39}$ The risk of infection is 6.6 times higher with general anesthesia. ${ }^{40}$ Nausea and vomiting associated with the general anesthesia produce high virulent airborne particles which have serious deleterious effects compromising the respiratory system. Although symptomatic patients are the primary source of infection, asymptomatic subjects may also spread the disease and should not be neglected. ${ }^{41}$ Regional anesthesia is recommended over general anesthesia; nasal prongs are used for oxygen supply. Aeration of closed environments, appropriate use of PPE kits, frequent hand hygiene, masks for patients, and surface decontamination are mandatory. ${ }^{42}$

\section{Recommendations}

- Local/regional anesthesia with minimal or no sedation is preferred for upper and lower limb procedures.

- General anesthesia should be reserved for individuals who need the most. If performed, follow strict and efficient 


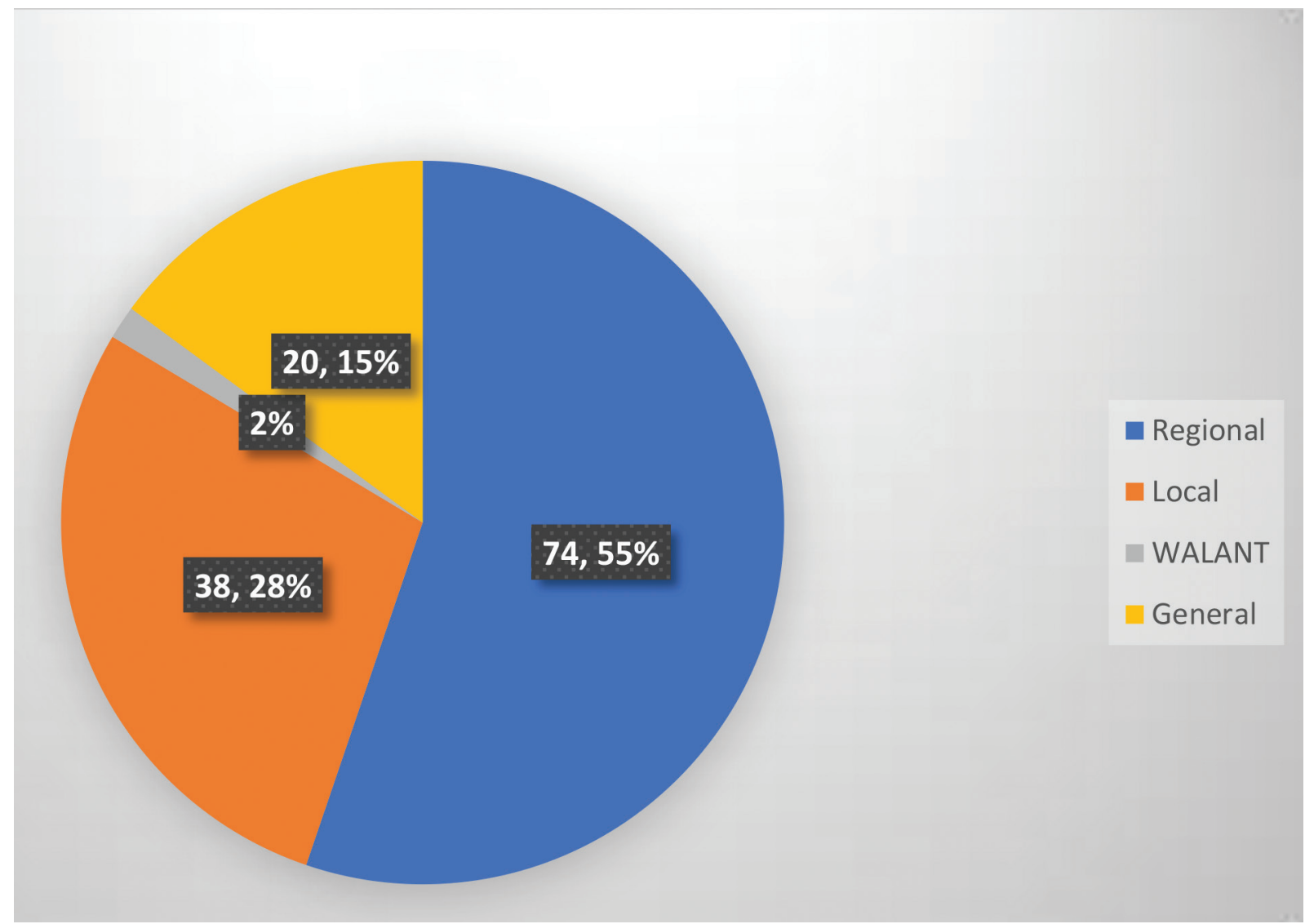

Fig. 13 Methods of anesthesia used in the study.

protocols to minimize aerosol droplet transmission. (PPE, N95 masks, 6-feet distancing from the patient's head, and postanesthesia care units are mandatory.)

- Patients should wear surgical masks throughout the procedure and if oxygen is required, it should be supplemented by nasal prongs.

- Minimize aerosolization, coughing, and circuit leaks.

Level of evidence: $\mathrm{V}$

Participants vote: agree: 91\%, disagree: 6\%, abstain: $3 \%$ (super majority, strong consensus) (95\% CI 84 to 95).

\section{Postoperative Care and Follow-Up}

Despite the current situations, 29\% of the surgeons still preferred to see the patients as usual on their working day with modifications in their OR and clinics, such as recovering in isolation rooms (disinfected with $70 \%$ ethanol, sodium hypochlorite, or $0.5 \%$ hydrogen peroxide), restricting visitors' entry, discharge as day care if possible, shifting to the ICU if needed, and using protective therapy protocols. Our analysis had day-care surgery and discharge by $20 \%$ surgeons and patients with extended stay were discharged in less than 3 days by $60 \%$ of the surgeons and 7 days by $9 \%$ of the surgeons.

Surgeons (9-31\%) restricted the postoperative follow-up to a particular day in a week or few days in a week. Fifteen percent of the surgeons preferred teleconsultation (video + telephone calls). Very limited procedures such as suture removal,
Kirschner wire removal, and splint and cast applications were done (22\%) on selected days on appointment basis. Subsequent follow-up consultations and regular patients' clinic visits strictly followed safe hygienic measures and social distancing measures such as outpatient/clinic patients (46\%) less than five patients per hour, seating arrangements of 2 meter distancing (75\%), 50:50 physical-digital consultations (13\%), restricting of nonessential materials (26\%; books, bags, etc.), and minimal contact and accompanying persons' entry (39\%). Some surgeons (31-34\%) preferred normal business schedule as usual like before (-Fig. 14).

\section{Rationale}

The human coronaviruses (e.g., SARS-CoV-2) can persist on inanimate surfaces in the OR which can be effectively inactivated by surface disinfection procedures, such as using $70 \%$ ethanol or $0.5 \%$ hydrogen peroxide. ${ }^{43-45}$ Besides, the OR can be cleaned with sodium hypochlorite followed by hydrogen peroxide vaporization as an added precautionary measure. ${ }^{46}$

Symptomatic patients or asymptomatic patients with COVID-19 test positive after the surgery should be kept isolated in the intensive ward of COVID with surgical masks. ${ }^{30}$ Theoretically, nonsteroidal anti-inflammatory drugs can potentially worsen symptoms in patients with COVID-1947 because of their increased expression of angiotensin-converting enzyme 2 (ACE-2) which enables SARS-CoV-2 (the coronavirus responsible for COVID-19) to bind to its target cells in the lung. It is prudent to avoid this class of drugs 


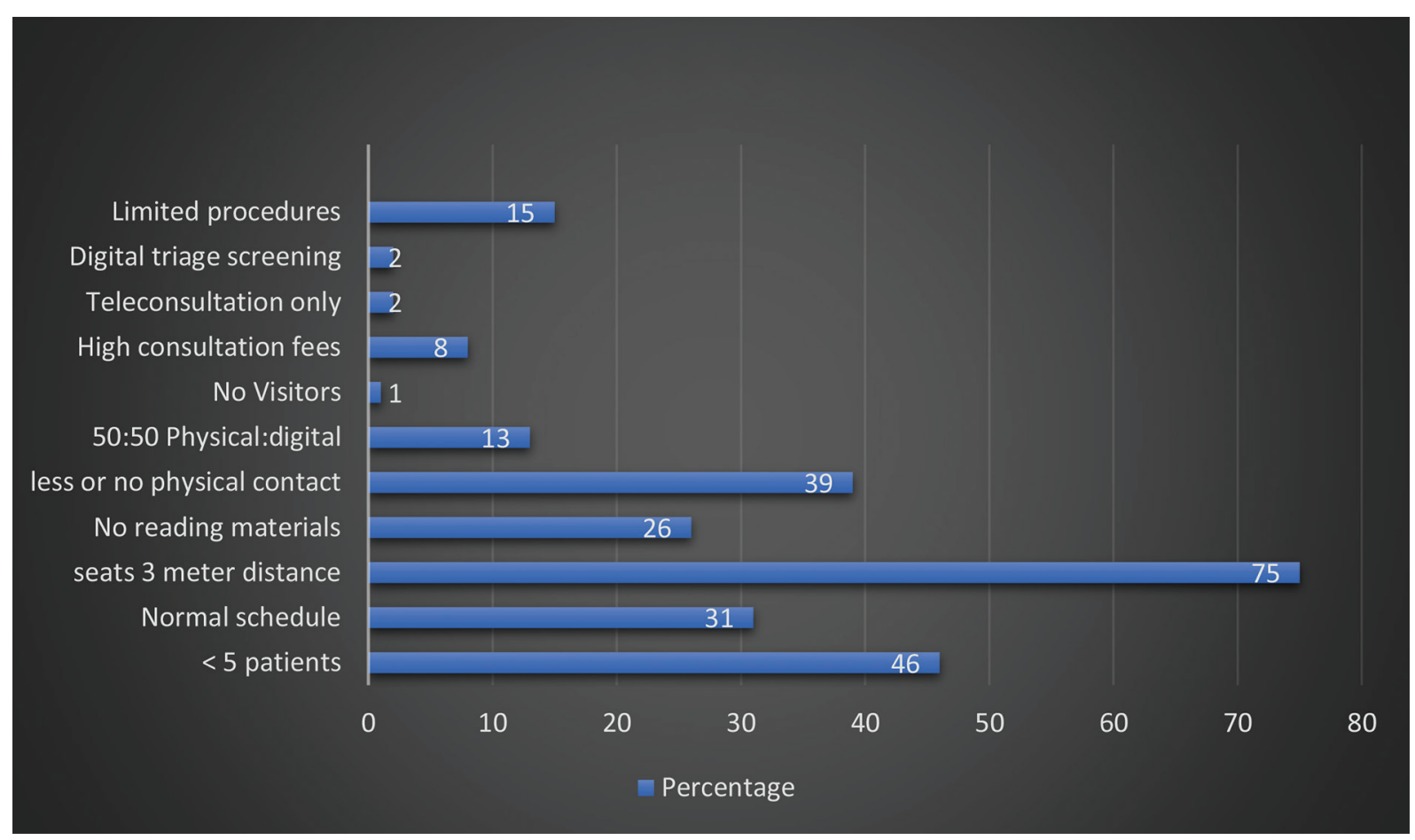

Fig. 14 Post-operative care and follow up.

and choose other classes of safe analgesics. A minimum of 1 hour should be planned between the cases to allow the OR to be properly decontaminated with all its equipment.

The face-to-face visits should be limited to urgent cases and selected postoperative care, which include postoperative COVID-19 symptoms, suture removal, evaluation of fracture reduction, and wound dressings. ${ }^{48}$ All patients should perform frequent and accurate hand hygiene and wear surgical masks inside the hospital premises, and health care providers should wear PPE kits, nonsterile gloves, face shields, N95/FFP3 respirators or surgical masks if unavailable, and protective glass face shields for droplet precautions. ${ }^{49}$ Telemedicine (videoconferencing) is a useful adjunct to conduct nonurgent follow-up and it provides rapid direct visualization of the patients. ${ }^{50}$ Besides it enhances telerehabilitation ${ }^{51}$ by teaching them exercises periodically, analyzing the range of movements, and further therapy as per the need.

\section{Recommendations}

- Day care surgery and early discharge.

- Teleconsultations (video and audio calls) and telerehabilitation.

- In every follow-up visit, patients must wear a mask and be screened for fever/cough/breathlessness.

- Surgeons must wear appropriate PPE kits (a disposable gown, nonsterile gloves, a face shield or goggles, an FFP2/ FFP3/N95 respirator, or a surgical mask).

\section{Level of evidence: $\mathrm{V}$}

Participants vote: agree: 91\%, disagree: 6\%, abstain: 3\% (super majority, strong consensus) (95\% CI 84 to 95).

\section{COVID-19 Test Positive after Surgery}

For all emergency surgeries, the surgeons did COVID-19 test of the patients in the postoperative period and found negative in $87 \%$, and positive in $13 \%$ (range 5-15 cases). COVID-19-positive cases were intimated to the local authority, shifted to tertiary care/government medical college hospitals for further follow-up and management.

\section{Recommendations}

RT-PCR tests for all elective cases and emergency patients in the postoperative ward. If positive, shift to tertiary care/governmental hospitals for ICU care.

\section{Level of evidence: $\mathrm{V}$}

Participants vote: agree: $86 \%$, disagree: $14 \%$ (super majority, strong consensus) (95\% CI 78 to 91 ).

\section{CT-Scan Chest}

Seventy-seven percent of the surgeons did not recommend CT-scan chest for their patients irrespective of emergency and elective nature. Thirteen percent of surgeons did CT scan chest to find for COVID-19 lung features before surgery in all of their patients. Some surgeons (7-10\%) preferred it when the radiographs or RT-PCR results were inconclusive (-Fig. 15).

\section{Rationale}

So far, the pattern of ground-glass and consolidative pulmonary opacities, often with bilateral and peripheral lung distributions, has remained the chest CT hallmark of COVID-19 infection. ${ }^{52}$ Considering the possibility of false-negative PCR 


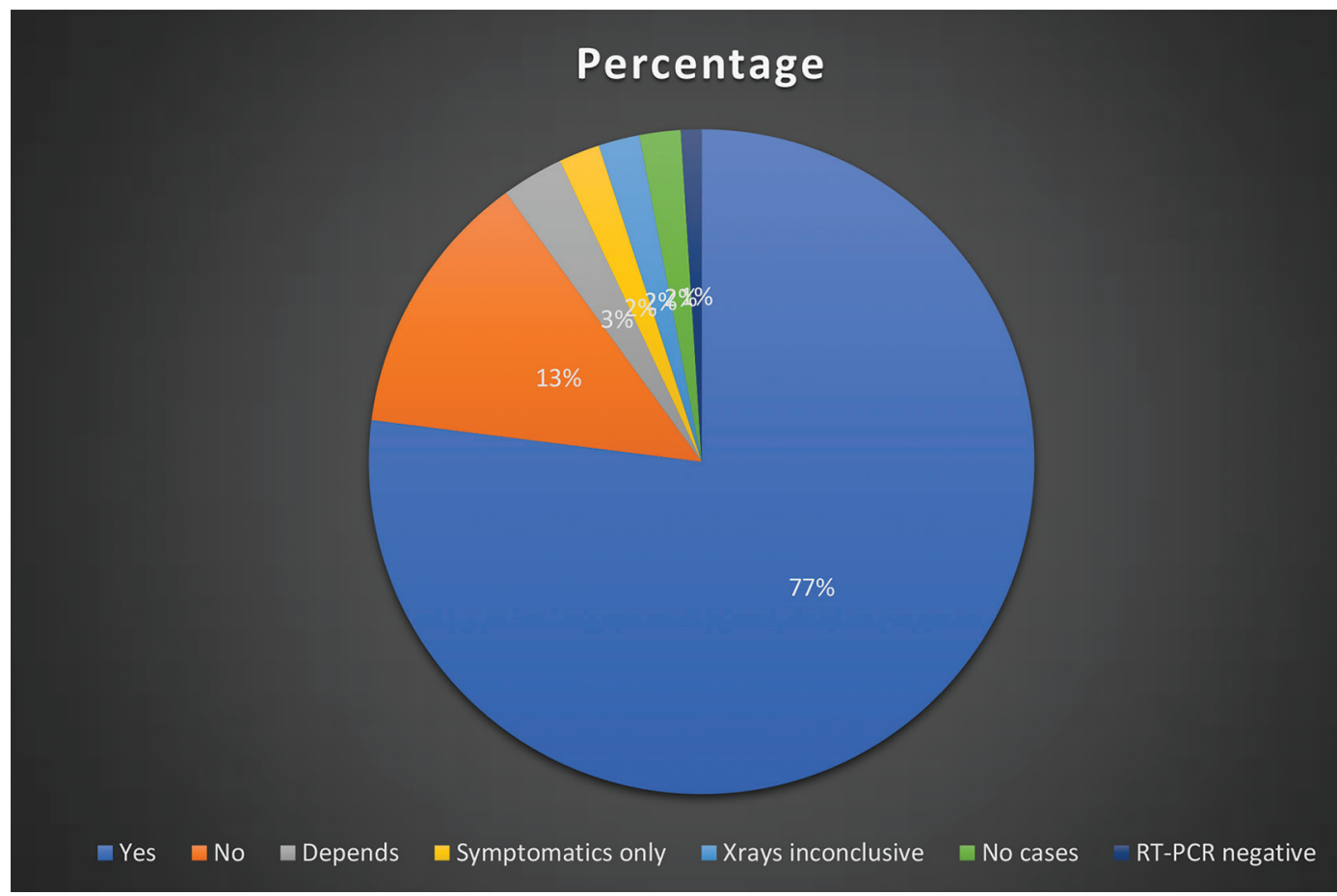

Fig. 15 Surgeons' recommendations for CT-scan chest.

Table 2 CT scan chest finding in COVID-19.

- Presence of ground-glass opacities
- Presence of consolidation
- Laterality of ground-glass opacities and consolidation
- Number of lobes affected where either ground-glass or
consolidative opacities were present
- Degree of involvement of each lung lobe in addition to
overall extent of lung involvement measured by means of a
"total severity score" as detailed below
- Presence of nodules
- Presence of a pleural effusion
- Presence of thoracic lymphadenopathy (defined as lymph
node size of $\geq 10$ mm in short-axis dimension)
- Airway abnormalities (including airway-wall thickening,
bronchiectasis, and endoluminal secretions
- Axial distribution of disease (categorized as no axial distri-
bution of disease, central "peribronchovascular" predomi-
- nant disease, or peripheral predominant disease)
fibrosis of underlying lung disease such as emphysema or

results, surgeons recommend diagnosis based on clinical and chest CT findings (ground glass opacities and consolidation). It is also agreed that COVID-19 has, in addition, different lung features and presentations similar to outbreaks such as SARS and Middle East respiratory syndrome (-Table 2). For symptomatic patients, CT scan is reliable when it is done 0 to 2 days after symptom onset and a delayed CT scan has limited sensitivity and negative predictive value.

\section{Recommendation}

CT scan findings such as consolidation, bilateral disease, greater total lung involvement, linear opacities, "reverse halo" sign, “crazy-paving” pattern, and peripheral lung distribution are found after confirmed COVID-19 infection. A routine scan may be required for specific cases.

Level of evidence: $\mathrm{V}$

Participants vote: agree: $49 \%$, disagree: $49 \%$, abstain: $2 \%$ (no majority, no consensus) (95\% CI 39 to 59).

\section{COVID-19 Tests}

All emergency surgeries included in the entire study were done without COVID-19 test and for all elective cases, surgeons kept COVID-19 test (RT-PCR) as mandatory. Still for emergency cases, $40 \%$ of the surgeons preferred the COVID19 test at the time of surgery or sooner in the postoperative period, which could help them to isolate asymptomatic but COVID-19-positive patients for further quarantine, treatment, and official reporting to the government agencies. For all elective cases and nonemergency cases, though RT-PCR testing was mandatory, only $77 \%$ of the surgeons did it in their cases and 14\% preferred immunoglobulin M (IgM) and immunoglobulin $\mathrm{G}(\operatorname{IgG})$ antibody testing. Lack of testing kits and initial hospital norms to compensate could be the reason. Some surgeons (10\%) relied on blood parameters (serum ferritin, interleukins, complete blood counts, C-reactive protein, 
liver functions test, leukocyte:neutrophil ratios, lymphocyte:neutrophil ratio) for their patients ( - Fig. 16).

\section{Rationale}

- Serological assays (IgG, IgM) for SARS-CoV-2 are done for identifying groups at high risk for COVID-19 infections.

- Real-time RT-PCR assay is a molecular diagnostic test. The samples from the nasopharyngeal swab have been widely used to detect SARS-CoV-2. This nucleic acid amplification test (NAAT) identifies genes specific for screening and confirming the disease. PCR amplification of the viral $E$ gene is a screening test and amplification of the RdRp region of the orf $1 \mathrm{~b}$ gene is a confirmatory test of RT-PCR for COVID-19.

There are several RT-PCR protocols ${ }^{53}$ for the detection of SARS-CoV-2 RNA posted by the WHO at https://www.cdc.gov/ coronavirus/2019-ncov/lab/resources/antibody-tests-guidelines.html (updated May 25, 2020 and accessed on May 28, 2020). Testing for IgG and IgM antibodies confirmed the persons' infection. In general, IgM is one of the first types of antibodies produced after infection and is most useful for determining recent infection, while IgG generally develops after IgM and may remain detectable for months or years. ${ }^{53}$

\section{Recommendations}

- Specimens should be collected from nasopharyngeal swab (preferred) and oropharyngeal swab. If not possible then collect from nasopharyngeal aspirates and sputum (if produced) and/or endotracheal aspirate or bronchoalveolar lavage in patients with more severe respiratory disease.
- Nucleic acid testing (NAAT) is the primary method of diagnosing COVID-19, which is based on the detection of unique sequences of virus RNA. Real-time RT-PCR detects SARS-CoV-2 genetically.

- PCR testing of asymptomatic or mildly symptomatic contacts can be considered in the assessment of individuals who have had contact with a COVID-19 case.

- Screening protocols should be adapted to the local situation. Even the transmission electron microscopy can identify the morphology of the virus (corona-shaped).

- No identified advantage of IgG and IgM antibody assays. Antibodies detected in the patient suggest that infection has occurred and some degree of immunity from infection has developed.

- Surgeons are also aware of false negatives from test reports and hope that advancement in NAAT techniques (e.g., CB NAAT [cartridge-based NAAT]) could possibly reduce the false negativity and open a window for clarity and focus.

\section{Level of evidence: $\mathrm{V}$}

Participants vote: agree: $100 \%$, disagree: 0 (super majority, strong consensus).

\section{Emergency Room and Pneumonia Ward Management}

Junior residents and registrars (21\%), junior consultants (38\%), and senior consultants (43\%) first received the orthopaedics emergencies in the ER, assessed, and operated them. Several countries (43\%) have COVID-19 triage protocols and separated the cases from normal health care services to prevent the risk of contamination. Involvement of junior residents in COVID-19 wards and removing them from their routine orthopaedics duties inevitably interrupt the learning and put them more prone to risk of infection.

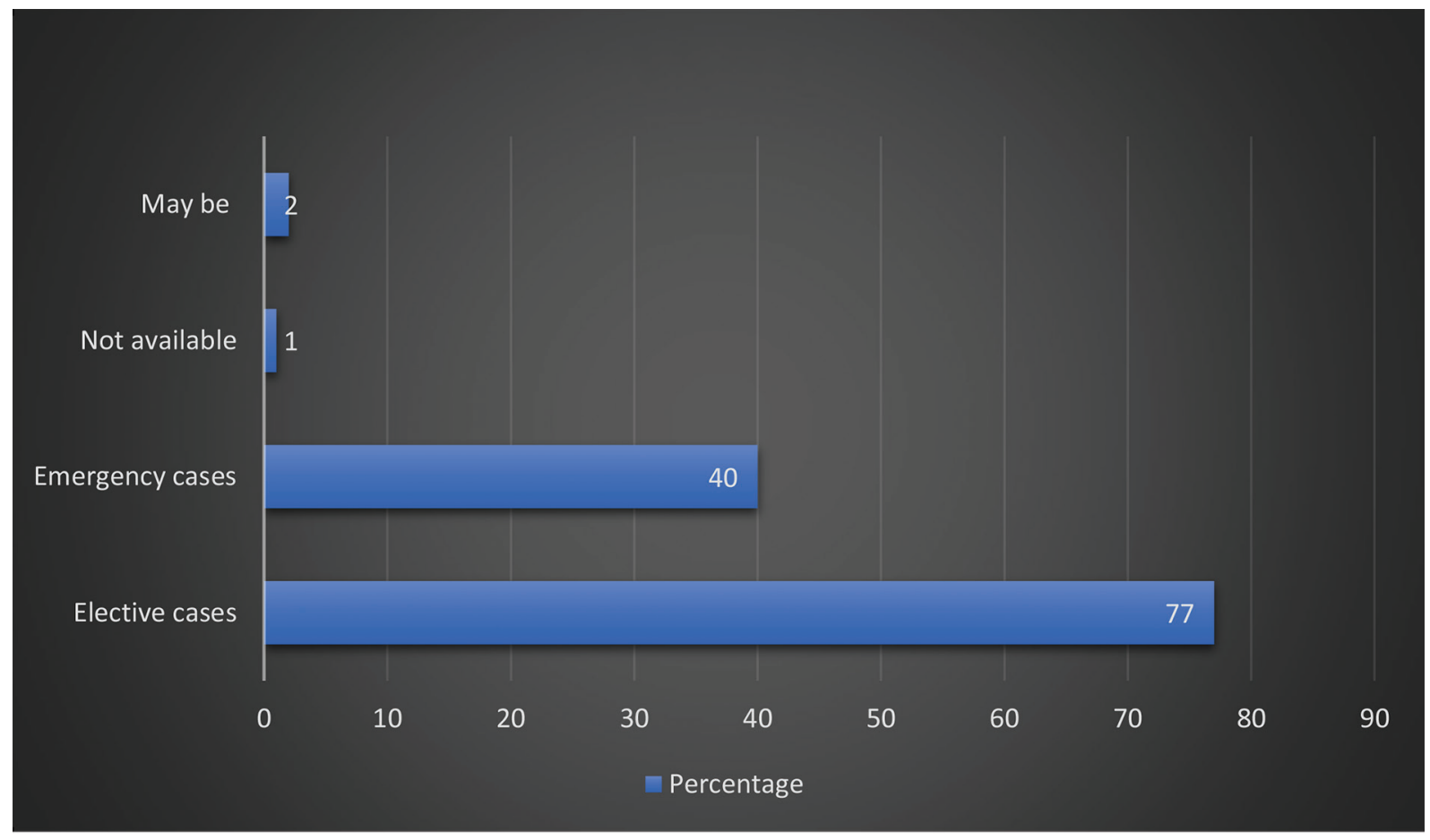

Fig. 16 Surgeons' recommendations for COVID-19 tests. 
Countries with national public health care systems and government medical colleges have their orthopaedics surgeons and allied specialists (30\%) posted in the pneumonia ward. By this, the patient load (asymptomatic and minimally affected patients) of the physicians and pulmonologists is reduced. Absence of active teaching of the trainees and orthopaedics surgical training for residents has become a reality. Specialists such as hand and microsurgeons were unavailable when really required for their emergencies and technically demanding surgeries.

\section{Rationale}

The overall goal during the fight against COVID-19 is to create a system model that allows for appropriate resource allocation (surgeons, specialists, staff, technicians, ICU staff, paramedics, and other health care workers) to provide optimal patient care, while minimizing viral spread to the patients and other members of the treatment team. ${ }^{54}$ Health care workers involved in the COVID-19 ward or ICU treatments may remain asymptomatic for up to 14 days. ${ }^{55}$ It is prudent and rational to have different teams of surgeons, staffs, and paramedical technicians to work on rosters so that each team can be 14 days away from the work.

\section{Recommendations}

Cyclical quarantine among the health care workers and COVID-19 test for suspected team members.

Level of evidence: $\mathrm{V}$

Participants vote: agree: 94\%, disagree: 6\% (super majority, strong consensus) (95\% CI 88 to 97 ).

\section{Shortage of Staff and Hospital Supplies}

Immediately after the lockdown, hospitals across the globe faced acute shortage of staff (25\%), nurses for the ward, ORs, and ICUs because of the transport restriction and initial fear among people about the dreadful virus. In order to meet the initial increasing demands, PPEs, surgical masks/N95/FFP3, and gowns (41\%) and medicines (7\%) fell short of supply. No free availability of surgical masks concerned $10 \%$ of surgeons involved in the study. Mostly, all national health care systems/government medical college hospitals in developed and developing countries jolted during the initial phase, came to stand still in $11 \%$ countries, and recuperated gradually with strict lockdown rules and procurement of PPE kits, masks, and ICU instruments (ventilators, monitors, and COVID-19 test kits; -Fig. 17).

Apart from government medical college hospitals and national public health care systems, $70 \%$ of private hospitals/

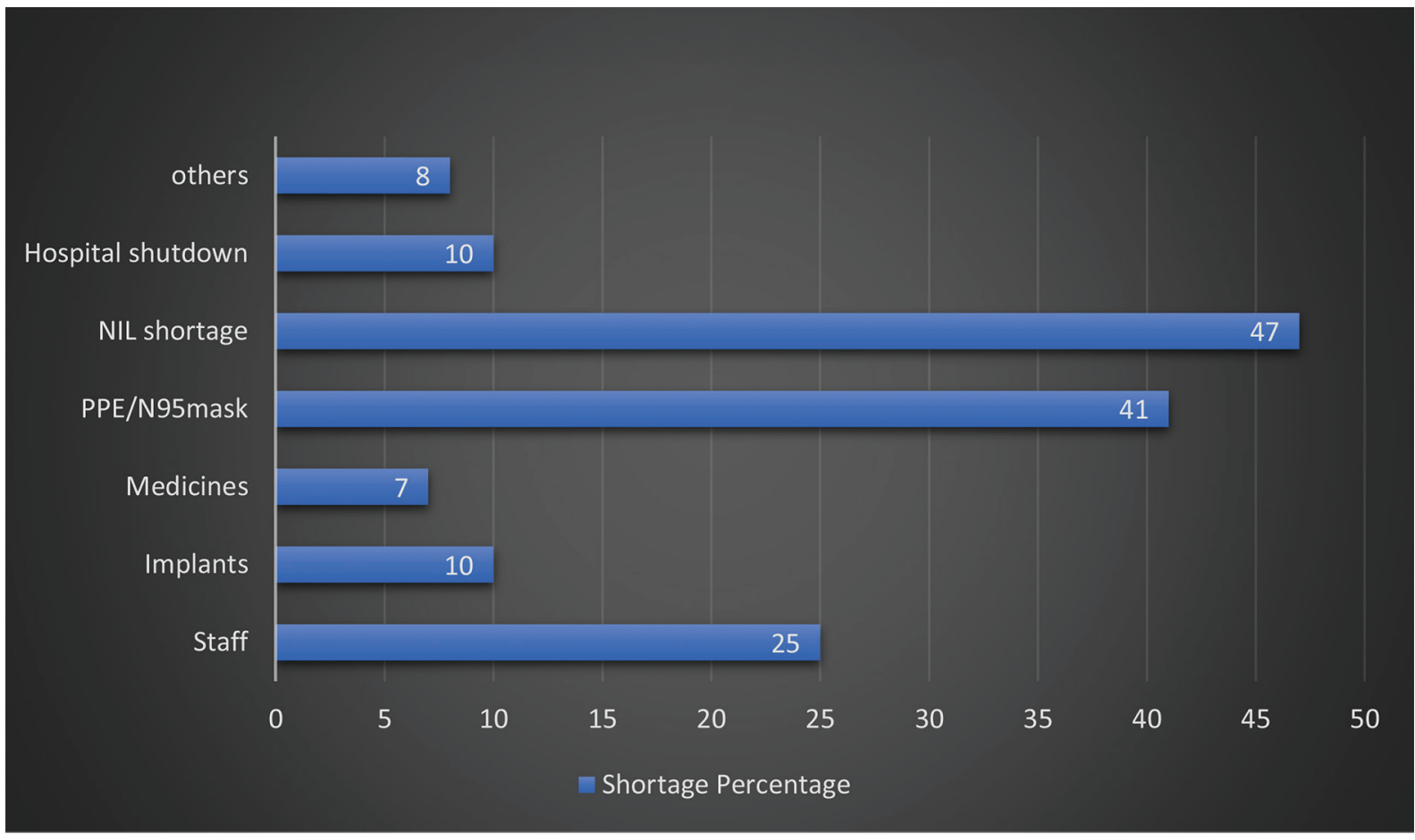

Fig. 17 Shortage of staff and hospital supplies. 
nursing homes had suffered a major setback financially; the shortage of manpower forced them to shut down the essential services. Many such hospitals have to furlough their staffs to 25 to $50 \%$ and paramedics to $50 \%$ of their normal strength and incurred financial constrains varying from 25 to $100 \%$ during the lockdown. Doctors and staff employed in the private health sectors faced 50 to $100 \%$ salary loss.

\section{Expectation to Normalcy Soon after the Lifting of Lockdown}

The suspension of elective surgeries put severe financial strains on the patients, physicians, and the entire health care system in all countries. One-third of the surgeons expected the current situation to improve in 15 days and $24 \%$ in 2 months' time. Surgeons (8.5\%) who were maintaining their operating schedule and clinic visits as usual during the lockdown continued the same after the official lockdown lift, and found no difference in the normalcy. Very few surgeons (5\%) worried about the future, kept fingers crossed, and waited for changes to happen soon. There are several factors which determine the return to normalcy, which include government policy of lifting lockdown, containment of the virus, flattening the curve, comprehensive protocol analysis based on countries' local need, and the infrastructure to bring back to normalcy if not fast but slowly and steadily ( - Fig. 18).

\section{Rationale}

Ceasing elective procedures and other services (master health check-up, etc.) in many hospitals has decreased their revenue and increased their expenses and daily working costs. The cash source was depleted, and the hospital operations were halted. ${ }^{56}$ The surgeons would prefer "cherry-picking" and "lemon dropping" surgical procedures avoiding the complex and less revenue surgeries. ${ }^{57}$ Since there is a negative economy and financial loss in the private sectors, the compensatory way would be to increase the cost for all surgical procedures and services rendered to the patients. As the medical systems look ahead to resume their elective cases, they require abundant support from the local government and insurance providers, and ways of quick settlements to the hospital. While writing this article, many challenging logistic issues came to the fore that one needs to answer, such as how much and how long to retain employees and staffs, cuts in salary and regular benefits, loan repayments, and hospital maintenance charges. These are crucial in getting private health care providers back to

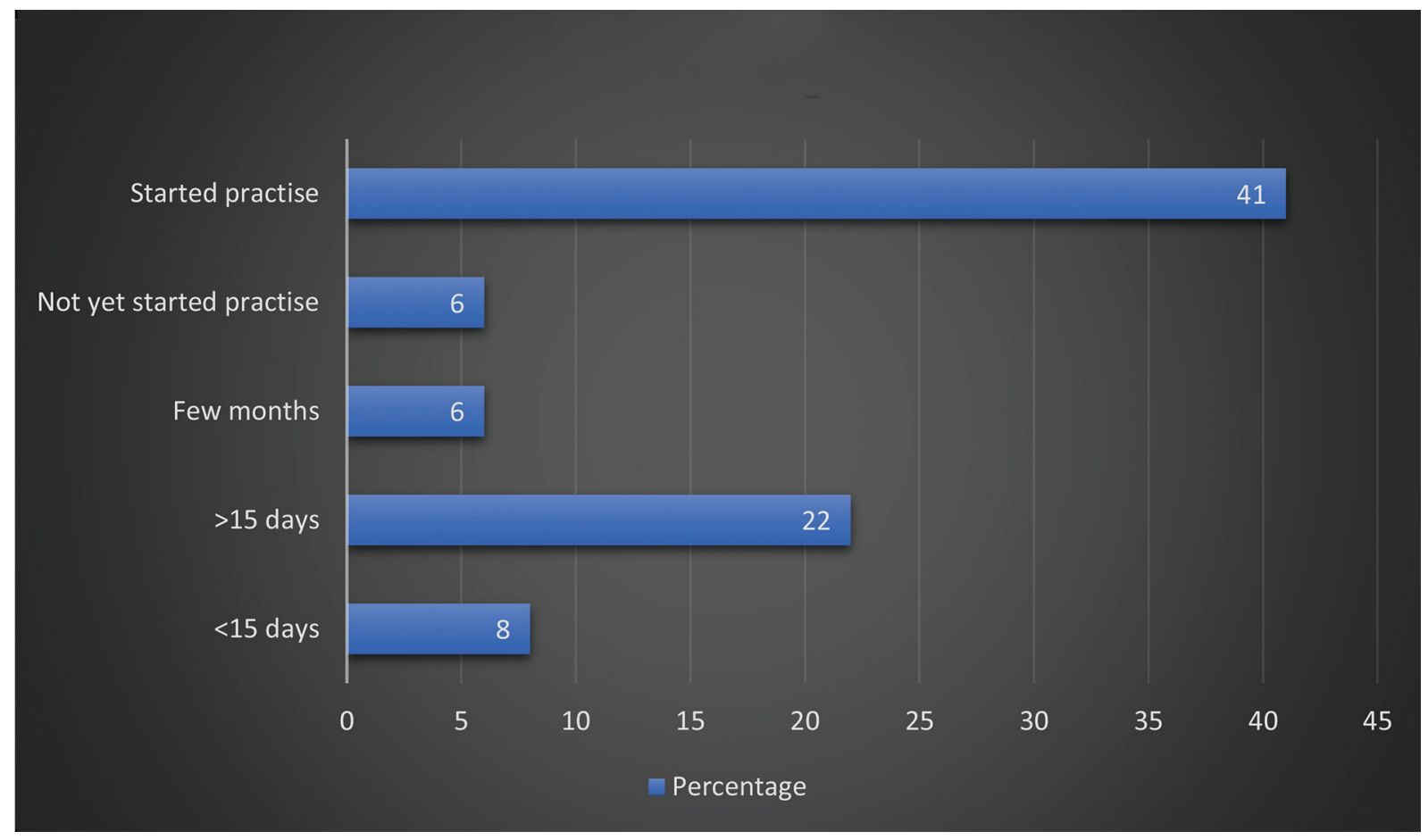

Fig. 18 Expectation to normalcy soon after the lift of lockdown. 
normalcy. ${ }^{57}$ Over the past decade, considerations were moving forward to ambulatory surgery centers ${ }^{58}$ and outpatient sitting arthroplasties. ${ }^{59}$ Multiple systemic reviews have reported these methods as safe and effective. ${ }^{60}$

\section{Recommendations}

- As both hospitals and patients are struggling to recuperate in this COVID-19 crisis, mutually benefitting strategic cost-effective and efficient surgical protocols supported by insurance providers and governments could make a big difference for normalcy to return.

- Proposing ambulatory surgery centers and outpatients' surgeries will disencumber the corona strain placed on hospital systems managing patients with COVID-19.

\section{Level of evidence: $\mathrm{V}$}

Participants vote:agree: 94\%, disagree: 6\% (super majority, strong consensus) (95\% CI 88 to 97 ).

\section{Orthopaedic Surgeons Are No More Surgeons}

Focusing their attention toward administrative works (26\%), writing, reviewing manuscripts (69\%), attending their household works, sharing their views and knowledge through online webinars (65\%), and making shifts in working made surgeons no more a surgeon in the lockdown period (-Fig. 19).

\section{Orthopaedic Surgeon Is No More Special}

Despite the fact that disclosure can be voluntary, $8 \%$ orthopaedic surgeons of different countries got infected (positive COVID-19 test) and 5\% tested were found negative during the lockdown. Out of fear, 15\% stopped operating and visiting clinics and remained confined in the home. Altruistically, $30 \%$ felt the noble service should be extended for emergency cases only and stop nonemergent clinics and surgeries.

\section{PPE Kits and Real Safety Concerns}

Fifteen percent surgeons believed the rationality behind the use of PPE kits during the crisis and felt 100\% safe, whereas 24\% surgeons felt half protected and 25\% felt $75 \%$ of protection from the risk of viral transmission; $23 \%$ surgeons felt unsafe with PPE/N95/FFP3 kits despite the scientific claim and proven facts ( - Fig. 20).

\section{Rationale}

Respirator masks (N95/FFP3) show protection factors 11.5 to 15.9 times greater than those of surgical masks. ${ }^{23}$ Moreover, a perfect-size and well-fitted mask leads to efficient sealing of the respiratory tract. Intact masks can be worn up to 8 hours continuously. ${ }^{23}$ European and U.S. standards for masks used for PPE showed that FFP1 has $80 \%$ filtration efficiency for particles of $0.3 \mu \mathrm{m}$, N95 (FFP2) has 95\%, and N99 (FFP3) has 99\%, offering good

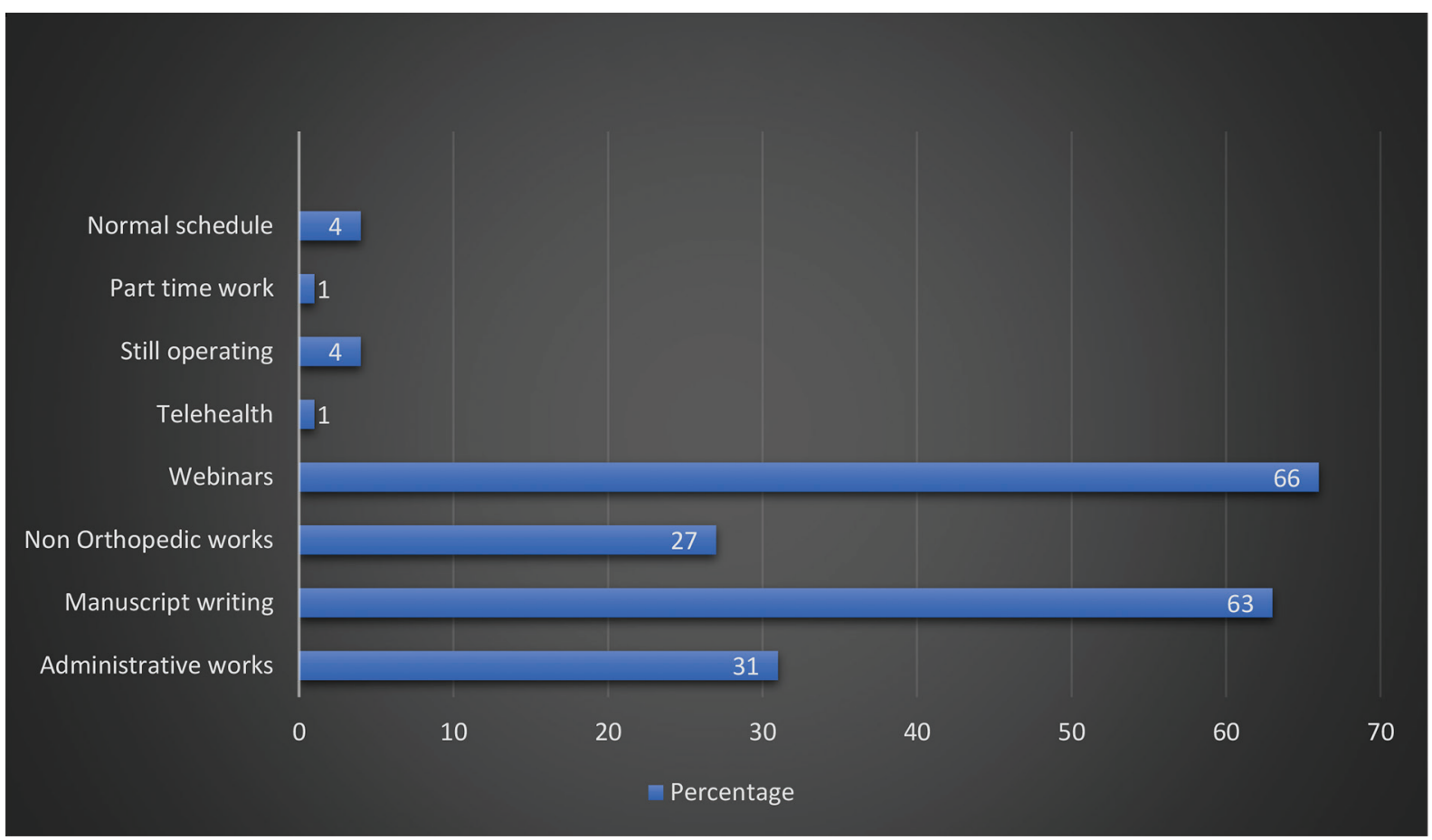

Fig. 19 Pattern of non-orthopeadics works during the lockdown. 


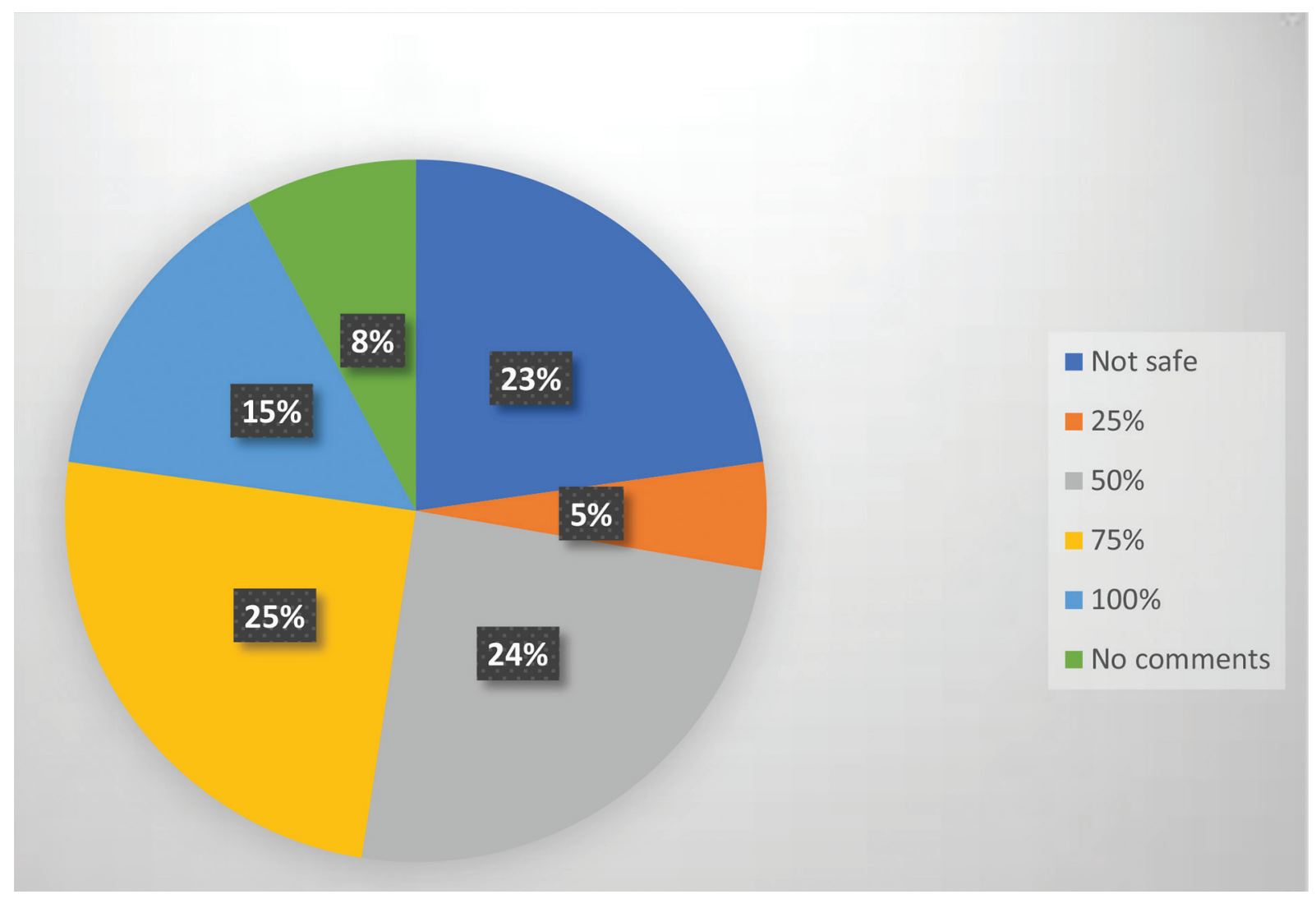

Fig. 20 How safe is PPE kits?

protection against airborne transmission. ${ }^{34}$ The European FFP3, equivalent to the U.S. N99, is recommended for aerosol protection against COVID-19. PPE is essential to prevent risk of transmission and cross-infections. A surgical gown which comprises the front area of the gown from chest to knees and the sleeves from the cuff to above the elbow has safety levels 1 to $4 .{ }^{21}$

\section{Recommendations}

- Respiratory AGPs, surgeries with high-speed devices (drill, saw, irrigations, etc.), extubating, and ending of anesthesia in the OR require FFP3 masks or powered air-purifying respirators, and face shields or surgical hoods, whereas surgical AGPs only require FFP2 masks.

- Level 4 surgical gowns, face shields or goggles, and double gloves should be used.

- In case of proven or suspected COVID-19 infection, orthopaedic surgeons should use FFP2-3 or N95-99 respirator masks.

- Cleaning of ORs should be done wearing FFP2/N95 or FFP3 masks.

- Caution for reuse and methods needed for reuse of N95 masks: 15 minute exposure to ultraviolet radiation, fumigation with hydrogen peroxide, hot water heating $\left(>56^{\circ} \mathrm{C}\right.$ [typically $60-80^{\circ} \mathrm{C}$ ] for 30 minutes followed by drying with a hair dryer), steaming (30 minutes of pressurized steam at $\left.121^{\circ} \mathrm{C}\right)$, and baking $\left(75^{\circ} \mathrm{C}\right.$ for 30 minutes).

Level of evidence: $\mathrm{V}$

Participants vote: agree: 94\%, disagree: 6\% (super majority, strong consensus) (95\% Cl 88 to 97).

\section{Surgeons Are Men of Fear}

Fifty-nine percent of the surgeons feared of getting infected and $28 \%$ considered them vulnerable to get infected. One surgeon $(1 \%)$ was getting treatment for their pneumonia associated with COVID-19 while writing this article. At this juncture, we all wish them a speedy recovery and safe return to work soon ( - Fig. 21).

Instantly, $80 \%$ of the surgeons feared of their near and dear ones getting infected from them. For which, they had self-quarantined in a separate room/part of the home, facing all odds and obstacles and sacrificing for their love ones.

\section{Rationale}

Transmission of COVID-19 can happen in 25\% of the orthopaedic surgeons. ${ }^{2}$ There were confirmed (20.8\%) transmissions of COVID-19 to family members, which always make orthopaedic surgeons feel panicked, depressed, and worried. 


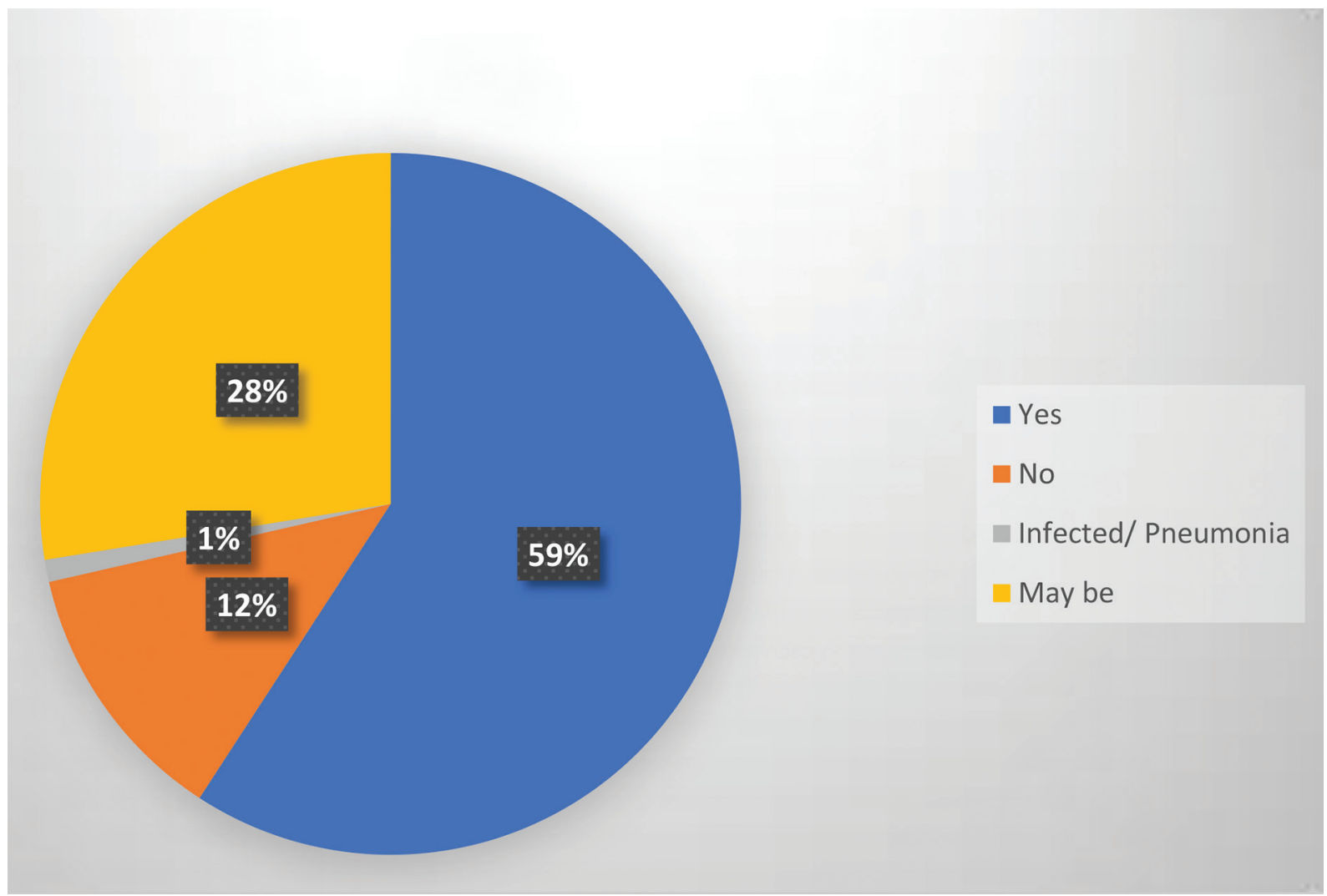

Fig. 21 Fear of infections/self infections

\section{Recommendations}

- In the case of COVID-19 positive, isolation of orthopaedic surgeons needs self-quarantine and avoid close contact with family members for 2 weeks.

- They can be released when there are complete resolutions of symptoms (temperature returns to normal for more than 3 days, respiratory symptoms are significantly relieved) RT PCR and antibody test is negative on two consecutive occasions (sampling interval $\geq 24$ hours).

\section{Level of evidence: IV}

Participants vote: agree: 94\%, disagree: 6\% (super majority, strong consensus).

\section{Orthopaedic Surgeons Are Good Chefs and Readers}

Spending lots of time with the wife and kids (44\%), helping them in the kitchen (42\%), and cleaning and home making (56\%) proved that orthopaedic surgeons were the best for their better half. Reading books and journals (70\%), watching movies and entertainment channels (48\%), yoga (8\%), going to gyms and bodybuilding (9\%), playing golf, gardening, and playing with the kids were the activities they did to spend their quality time during the lockdown ( - Fig. 22).

\section{Surgeons Are Not Sprinters}

Treadmills (22\%), cross trainers (8\%), Zumba (3\%), You tube exercises (15\%), workouts, jogging, bike riding, limited outdoor running, and meditations were performed during the lockdown as their cardiac and physical fitness measures. More than 50\% stayed within the home, fearing not to get injured while running/jogging, but utilized the best available time in their interests.

\section{Orthopaedics Surgery and Allied Subspecialty Meetings}

Fifty-nine percent of the surgeons favored virtual webinars and limited meetings, and $12 \%$ favored webinars only. Interestingly, $23 \%$ of the surgeons prefer normal meetings at the conference venues because they take pleasure in face-to-face meet and like the real-time happenings. Zoom Tm (66\%), webinars (40\%), Facetime (16\%), Skype Tm (20\%), Google Chat/Duo Tm (17\%), WhatsApp videos Tm, Microsoft Teams Tm, Hospital software, and Halo app were the preferred tools for their virtual meetings during the lockdown ( - Fig. 23).

\section{Rationale}

Virtual learning through webinars, meetings, and online lectures helps participants in various ways; they can record and reproduce the events, can access and share images, and feel at ease in attending during the work and other schedules. ${ }^{54}$ It has no personal face-to-face contact, economically productive, and more viable than the real conferences. ${ }^{61}$ Video-based education promotes surgical training by providing audiovisual contents on indications, preoperative work-up, OR settings, operative techniques, and postoperative care. ${ }^{62}$ 


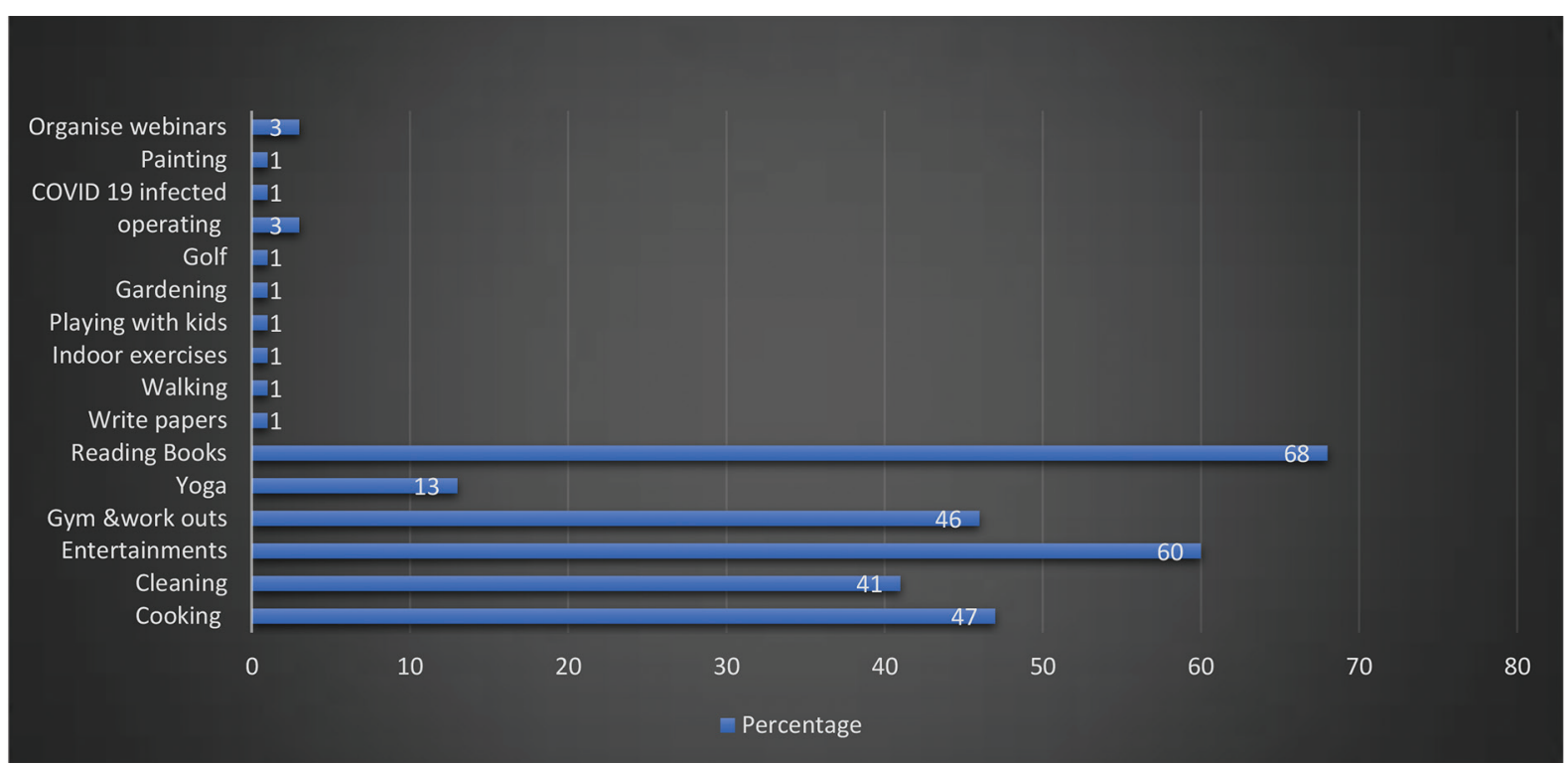

Fig. 22 Utilization of time apart from normal schedule during the lock down.

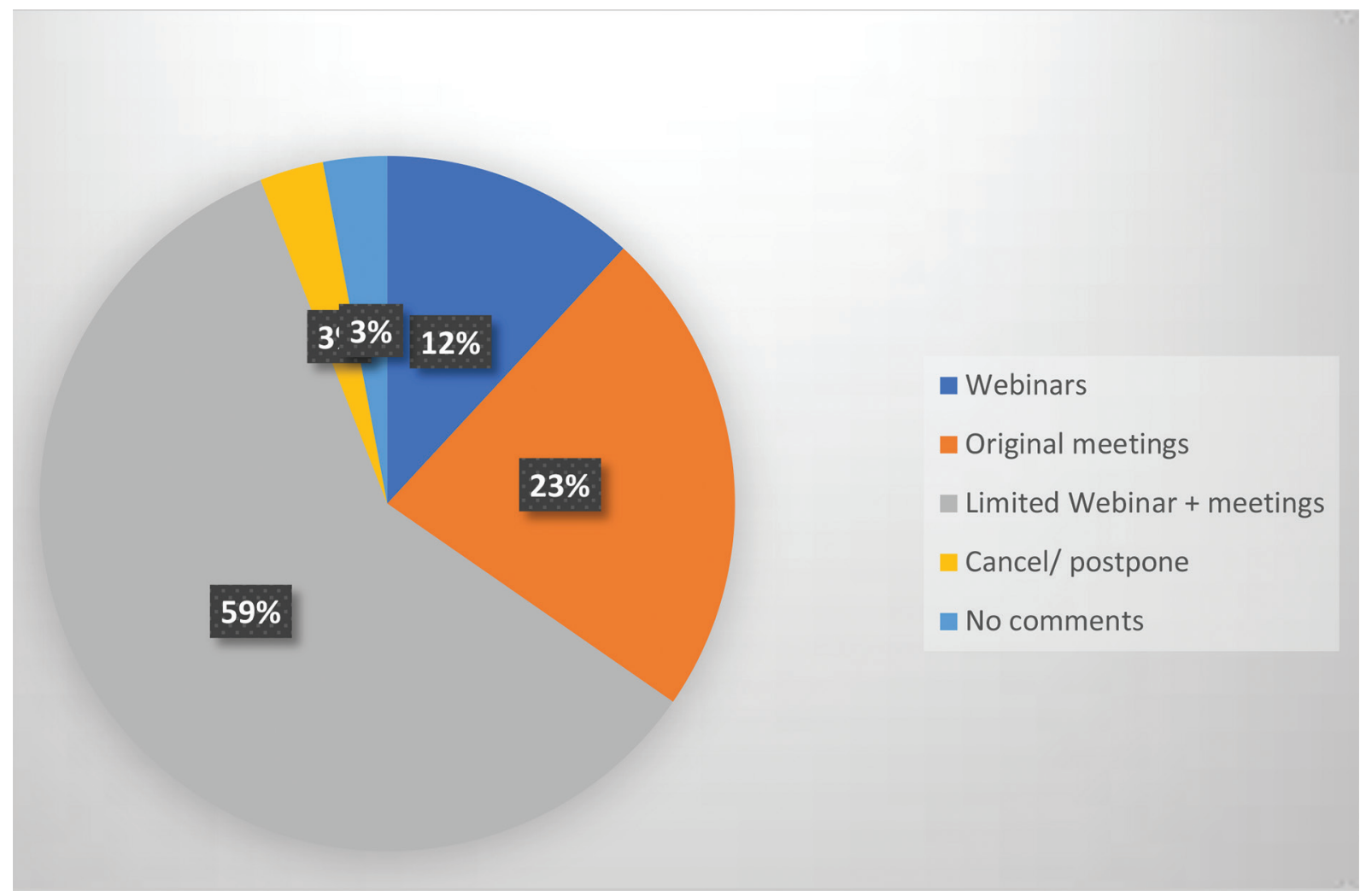

Fig. 23 Orthopedics surgery and allied subspecialty meetings.

\section{Recommendations}

Limited meetings and online webinars are useful resources to implement surgical education in the COVID-19 era.

\section{Level of evidence: $\mathrm{V}$}

Participants vote:agree: 94\%, disagree: 6\% (super majority, strong consensus) (95\% CI 88 to 97).

\section{Ethical Considerations}

Although teleconsultation and virtual meetings have been much improvised to provide better health care, the physician-patient relationship which has been the existing norm for millennia, such as meet and greet, gentle and tender examinations, and shared decision-making, seems to have vanished in the COVID-19 era. 
Surgeons who were saviors in eradicating pain and disability have now become warriors against the pandemic disease. He may not be allowed to do his best for his patients and at times he may be compelled to say no to many things in his practice and incontrovertibly treat few of them conservatively. COVID-19 has put the orthopaedic surgeons in a censorious and interpretive position where they have to protect themselves and their family members from viral transmissions and at the same time have to actively indulge in his profession. Few of the surgeons have been terminated from the job and their positions, which imposed significant psychological and financial burdens.

\section{Mandatory Check in for Orthopaedic Surgeons}

- Awareness of the current and projected COVID-19 cases in the local community and country.

- Regular and frequent reporting to the concern local authority.

- Making an adequate supply of PPE, medical gases, drugs, and essential stocks available at the practice location and in the country.

- Staffing and paramedical technicians' availability. Duty roster and shifts.

- Medical officers/ambulatory service location capacity.

- Testing centers in the local community/region.

- Health and age of each individual patient and their risk for severe disease.

Level of evidence: $\mathrm{V}$

Participants vote:agree: 97\%, disagree: 3\% (super majority, strong consensus) (95\% Cl 92 to 99).

\section{Protecting Yourself and Others from the Spread of COVID-19}

- Regularly and thoroughly clean your hands with an alcohol-based hand rub or wash them with soap and water.

- Maintain at least 1 meter (3 feet) distance between yourself and others. Avoid going to crowded places.

- Avoid touching eyes, nose, and mouth. Make sure you and the people around you follow good respiratory hygiene. This means covering your mouth and nose with your bent elbow or tissue when you cough or sneeze. Then dispose of the used tissue immediately and wash your hands.

- Stay home and self-isolate even with minor symptoms such as cough, headache, and mild fever, until you recover. Have someone bring you supplies. If you need to leave your house, wear a mask to avoid infecting others.

- If you have fever, cough, and difficulty breathing, seek medical attention, but call by telephone in advance if possible and follow the directions of your local health authority.

- Stay up to date on the latest information from trusted sources, such as WHO or your local and national health authorities.
Level of evidence: $\mathrm{V}$

Participants vote: agree: 97\%, disagree: 3\% (super majority, strong consensus) (95\% CI 92 to 99).

\section{Discussions}

The surgeons have uniform opinion that everyone somehow will be infected at a certain point of time and 80 to $90 \%$ will develop mild-to-moderate symptoms or remain asymptomatic until they all develop herd immunity. Among the vulnerable people of getting infected, fractures and injuries do need more attention and care. Every orthopaedic surgeon has a dual role in his life-surgeon and husband or an individual. As a surgeon he intends to seek updates on the current recommendations in treating fractures and injuries during COVID-19 crisis. Whether to treat conservatively or operate the patients during the pandemic crisis needs certain guidelines and global consensus.

This article outlines the basic, fundamental, and consensual treatment protocols for orthopaedic surgeons. These recommendations are based on scientific research analysis and consensual agreement between 100 surgeons from 50 countries in the world who are dealing COVID-19 actively. As we all know change is life and changes have to happen. The protocols may change over the period of time depending upon the virus resurgence or viral mutations with different presentations or even with its end with simple herd immunity in the community. In the meantime, these recommendations and guidelines will make a perceptible change in the practice of orthopaedic surgeons. As a husband or an individual in the community, he plays a responsible role and a role model through active participation during the pandemic and self-quarantining to prevent the viral spread to his family and the living community.

International consensus and recommendations for safe orthopaedics surgical practice start from delineating the symptomatic from asymptomatic patients by giving special attention to the travel history, contact history, and screening of the elective cases with RT-PCR and in very essential cases with CT-scan chest. All emergency procedures shall be done at the earliest with full precautionary measures (PPE kits, N95/FFP3 masks, face shields, protective glass, footwear, and gowns). Once the patients are segregated, the orthopaedic surgeon judiciously decides the treatment plan based on the acuity level discussed in this article. Low-acuity patients are treated conservatively, and intermediate- and high-acuity patients are to be treated surgically. If negative pressure ventilations or safe ORs with limited manpower are available, a simple, definitive, and safe operative procedure should be performed. Day care surgery is preferred and in the case of extended stay, surgeons should plan for early discharge and follow-up. Full PPE kits with protective accessories should be available for surgeons for the clinics and ORs, and the patients should wear surgical masks throughout the stay in the hospital. Limited follow-up, teleconsultation, and telerehabilitation 
will prevent the face-to-face contact and the viral transmission. Safe practice at the hospital and home saves one from contaminating the family and community. Virtual meetings, webinars, journals, manuscript writings, exercise, and home making could be a break in orthopaedics surgeons' life. Proper planning and investments could prevent the financial loss at this time. Be in a pneumonia ward or in an OR in the home, orthopaedic surgeons are one of the real heroes fighting against the COVID-19 pandemic. There are many limiting factors in the study. Given the wide geographical variation, socio-economic status, and climate differences, random selection of surgeons of various employment statuses (private/government) from 50 countries to form a consensual recommendation needs comprehensible understanding and balance for the readers. Despite all these constraints, the current recommendations are rational and literature-supported.

All authors agreed that:

- They have no competing interest.

- They did not receive any grant from funding agencies in the public, commercial, or not-for-profit sectors.

- They understood and met the current International Committee of Medical Journal Editors (ICMJE) criteria for authorship.

- The survey has not involved any experimentation on humans or animals.

- The survey did not involve patients or volunteers.

\section{Note}

The views expressed in this article are those of the authors and do not represent the official policy of the any country.

\section{Conflict of Interest}

None declared.

\section{References}

1 Jerome JTJ. Is everything okay? COVID-19. J Hand Microsurg 2020. Doi: 10.1055/s-0040-1709948

2 Guo X, Wang J, Hu D, et al. Survey of COVID-19 disease among orthopaedic surgeons in Wuhan, People's Republic of China. J Bone Joint Surg Am 2020;102(10):847-854

3 WHO. Coronavirus disease (COVID-19) technical guidance: patient management. Available at: https://www.who.int/ emergencies/diseases/novel-coronavirus-2019/technical-guidance/patient-management. Accessed June 11, 2020

4 Centers for Disease Control and Prevention. Information for healthcare professionals about coronavirus (COVID-19). Available at: https://www.cdc.gov/coronavirus/2019-ncov/ healthcare-facilities/index.html. Accessed May 29, 2020

5 National Clinical Programme in Surgery. Information for surgeons regarding OPD triage during COVID-19 epidemic. Available at: https://msurgery.ie/wp-content/ uploads/2020/03/v3-NCPS-guidance-to-surgeons-for-OPDtriage-during-COVID-19.pdf. Accessed May 29, 2020

6 Centers for Disease Control and Prevention. Interim infection prevention and control recommendations for patients with suspected or confirmed coronavirus disease 2019 (COVID-19) in healthcare settings. Available at: https:// www.cdc.gov/coronavirus/2019-ncov/infection-control/ control-recommendations.html. Accessed May 29, 2020

7 Guy DK, Bosco JA, III, Savoie FH., III . AAOS guidelines for elective surgery during the COVID-19 pandemic. Available at: https://www.aaos.org/about/covid-19-information-for-ourmembers/aaos-guidelines-for-elective-surgery/. Accessed June 11, 2020

8 Chen YC, Lin WC. Risk of long-term infection-related death in clinical osteoporotic vertebral fractures: A hospital-based analysis. PLoS One 2017;12(8):e0182614

9 HIP ATTACK Investigators. Accelerated surgery versus standard care in hip fracture (HIP ATTACK): an international, randomised, controlled trial. Lancet 2020;395(10225):698-708

10 Lizaur-Utrilla A, Lopez-Prats FA. Hip attack for hip fractures: is ultra-early surgery necessary. ?Lancet 2020;395(10225): 661-662

11 Ravi B, Pincus D, Wasserstein D, et al. Association of overlapping surgery with increased risk for complications following hip surgery: a population-based, matched cohort study. JAMA Intern Med 2018;178(1):75-83

12 Wong KC, Leung KS, Hui M. Severe acute respiratory syndrome (SARS) in a geriatric patient with a hip fracture. A case report. J Bone Joint Surg Am 2003;85(7):1339-1342

13 Mi B, Chen L, Xiong Y, Xue H, Zhou W, Liu G. Characteristics and early prognosis of COVID-19 infection in fracture patients. J Bone Joint Surg Am 2020;102(9):750-758

14 Liu VX, Rosas E, Hwang J, et al. Enhanced recovery after surgery program implementation in 2 surgical populations in an integrated health care delivery system. JAMA Surg 2017;152(7):e171032

15 Pietrafesa CA, Hoffman JR. Traumatic dislocation of the hip. JAMA 1983;249(24):3342-3346

$16 \mathrm{Zh}$, en, g MH, Boni L, Fingerhut A. Minimally invasive surgery and the novel coronavirus outbreak: lessons learned in China and Italy. Ann Surg 2020. Doi: 10.1097/SLA.0000000000003924

17 Alleblas CC, Velthuis S, Nieboer TE, Sietses C, Stegeman DF. The physical workload of surgeons: a comparison of SILS and conventional laparoscopy. Surg Innov 2015;22(4):376-381

18 Janhofer DE, Lakhiani C, Song DH. Addressing surgeon fatigue: current understanding and strategies for mitigation. Plast Reconstr Surg 2019;144(4):693e-699e

19 McCormick F, Kadzielski J, Landrigan CP, Evans B, Herndon $\mathrm{JH}$, Rubash HE. Surgeon fatigue: a prospective analysis of the incidence, risk, and intervals of predicted fatigue-related impairment in residents. Arch Surg 2012;147(5):430-435

20 Nogler M, Lass-Flörl C, Wimmer C, Mayr E, Bach C, Ogon M. Contamination during removal of cement in revision hip arthroplasty. A cadaver study using ultrasound and highspeed cutters. J Bone Joint Surg Br 2003;85(3):436-439

21 American Society for Testing and Materials (ASTM) F2407. (2020). Available at: https://www.astm.org/COVID-19/. Accessed June 11, 2020

22 He X, Lau EHY, Wu P, et al. Temporal dynamics in viral shedding and transmissibility of COVID-19. Nat Med 2020;26(5):672-675

23 Lee SA, Hwang DC, Li HY, Tsai CF, Chen CW, Chen JK. Particle size-selective assessment of protection of European standard FFP respirators and surgical masks against particles-tested with human subjects. J Healthc Eng 2016;20168572493

24 Burnett RS, Berger RA, Paprosky WG. Della Valle CJ, Jacobs JJ, Rosenberg AG. Extensor mechanism allograft reconstruction after total knee arthroplasty. A comparison of two techniques. J Bone Joint Surg Am 2004;86(12):2694-2699

25 Ho, e Gan W, Wah Lim J, Koh D. Preventing intra-hospital infection and transmission of COVID-19 in healthcare workers. Saf Health Work 2020. Doi: 10.1016/j.shaw.2020.03.001 
26 NationalChildren's Medical Center \& Children's Hospital of Fudan University's Guideline Formulating Team of Rapid Screening \& Clinical Practices for Suspected and Confirmed Cases of Pediatric Novel Coronavirus Infection or Pneumonia Guideline of Rapid Screening \& Clinical Practices for Suspected and Confirmed Cases of Pediatric Novel Coronavirus Infection or Pneumonia. Chin J Evid Based Pediatr 2020;15:1-4

27 Rodrigues-Pinto R, Sousa R, Oliveira A. Preparing to perform trauma and orthopaedic surgery on patients with COVID-19. J Bone Joint Surg Am 2020;102(11):946-950

28 Chee VW, Khoo ML, Lee SF, Lai YC, Chin NM. Infection control measures for operative procedures in severe acute respiratory syndrome-related patients. Anesthesiology 2004;100(6):1394-1398

29 Wax RS, Christian MD. Practical recommendations for critical care and anesthesiology teams caring for novel coronavirus (2019-nCoV) patients. Can J Anaesth 2020;67(5):568-576

30 Ti LK, Ang LS, Foong TW, Ng BSW. What we do when a COVID-19 patient needs an operation: operating room preparation and guidance. Can J Anaesth 2020;67(6):756-758

31 Yeh HC, Turner RS, Jones RK, Muggenburg BA, Lundgren DL, Smith JP. Characterization of aerosols produced during surgical procedures in hospitals. Aerosol Sci Technol 1995;22:151-161

32 Tellier R, Li Y, Cowling BJ, Tang JW. Recognition of aerosol transmission of infectious agents: a commentary. BMC Infect Dis 2019;19(1):101

33 Heinsohn P, Jewett DL. Exposure to blood-containing aerosols in the operating room: a preliminary study. Am Ind Hyg Assoc J 1993;54(8):446-453

34 Hirschmann MT, Hart A, Henckel J, Sadoghi P, Seil R, Mouton C. COVID-19 coronavirus: recommended personal protective equipment for the orthopaedic and trauma surgeon. Knee Surg Sports Traumatol Arthrosc 2020;28(6):1690-1698

35 Liang ZC, Chong MSY, Sim MA, et al. Surgical considerations in patients with COVID-19: what orthopaedic surgeons should know. J Bone Joint Surg Am 2020;102(11):e50

36 Donaldson AJ, Thomson HE, Harper NJ, Kenny NW. Bone cement implantation syndrome. Br J Anaesth 2009;102(1):12-22

37 Högel F, Gerlach UV, Südkamp NP, Müller CA. Pulmonary fat embolism after reamed and unreamed nailing of femoral fractures. Injury 2010;41(12):1317-1322

38 Anfinrud P, Stadnytskyi V, Bax CE, Bax A. Visualizing speech-generated oral fluid droplets with laser light scattering. N Engl J Med 2020;382(21):2061-2063

39 WHO. Infection prevention and control during healthcare when COVID-19 is suspected 2020; Available at: https://www. who.int/publications-detail/infection-prevention-and-control-during-health-care-when-novel-coronavirus-(ncov)-infection-is-suspected-20200125. Accessed April 20, 2020

40 Tran K, Cimon K, Severn M, Pessoa-Silva CL, Conly J. Aerosol generating procedures and risk of transmission of acute respiratory infections to healthcare workers: a systematic review. PLoS One 2012;7(4):e35797

41 Bai Y, Yao L, Wei T, et al. Presumed asymptomatic carrier transmission of COVID-19. JAMA 2020;323(14):1406-1407

42 A, wa, d ME, Rumley JCL, Vazquez JA, Devine JG. Perioperative considerations in urgent surgical care of suspected and confirmed coronavirus disease 2019 orthopaedic patients: operating room protocols and recommendations in the current coronavirus disease 2019 pandemic. J Am Acad Orthop Surg 2020. Doi: 10.5435/JAAOS-D-20-00227

43 Kampf G, Todt D, Pfaender S, Steinmann E. Persistence of coronaviruses on inanimate surfaces and their inactivation with biocidal agents. J Hosp Infect 2020;104(3):246-251

44 Hulkower RL, Casanova LM, Rutala WA, Weber DJ, Sobsey MD. Inactivation of surrogate coronaviruses on hard surfaces by health care germicides. Am J Infect Control 2011;39(5):401-407

45 Kariwa H, Fujii N, Takashima I. Inactivation of SARS coronavirus by means of povidone-iodine, physical conditions and chemical reagents. Dermatology 2006;212(Suppl 1) :119-123

46 Pottage T, Richardson C, Parks S, Walker JT, Bennett AM. Evaluation of hydrogen peroxide gaseous disinfection systems to decontaminate viruses. J Hosp Infect 2010;74(1):55-61

47 Fang L, Karakiulakis G, Roth M. Are patients with hypertension and diabetes mellitus at increased risk for COVID-19 infection? Lancet Respir Med 2020;8(4):e21

48 St, inn, er DJ, Lebrun C, Hsu JR, Jahangir AA, Mir HR. The orthopaedic trauma service and COVID-19 - practice considerations to optimize outcomes and limit exposure. J Orthop Trauma 2020. Doi: 10.1097/ВOT.0000000000001782

49 Chang Liang Z, Wang W, Murphy D, Po Hui JH. Novel coronavirus and orthopaedic surgery: early experiences from Singapore. J Bone Joint Surg Am 2020;102(9):745-749

50 Loeb AE, Rao SS, Ficke JR, Morris CD, Riley LH III, Levin AS. Departmental experience and lessons learned with accelerated introduction of telemedicine during the COVID-19 crisis. J Am Acad Orthop Surg 2020;28(11):e469-e476

51 Prada C, Chang Y, Poolman R, Johal H, Bhandari M. Best practices for surgeons. COVID-19 evidence-based scoping review. A unifying report of global recommendations. 2020. Available at: https://myoe.blob.core.windows.net/docs/OE-Best-Practicesfor-Surgeons-COVID-19-Evidence-Based-Scoping-Review.pdf. Accessed June 11, 2020

52 Bernheim A, Mei X, Huang M, et al. Chest CT findings in coronavirus disease-19 (COVID-19): relationship to duration of infection. Radiology 2020;295(3):200463

53 CDC. Interim guidelines for COVID-19 antibody testing. Available at: https://www.cdc.gov/coronavirus/2019-ncov/ lab/resources/antibody-tests-guidelines.html. Accessed May 31,2020

54 Schwartz A, Wilson J, Boden S, et al. Managing resident workforce and education during the COVID-19 pandemic. J Bone Joint Surg Am 2020;5(2):e0045

55 Chavez S, Long B, Koyfman A, et al. Coronavirus disease (COVID-19): a primer for emergency physicians. Am J Emerg Med 2020, Doi: 10.1016/j.ajem.2020.03.036

56 Grimm CA. Hospital Experiences Responding to the COVID-19 Pandemic: Results of a National Pulse Survey March 23-27, 2020. Washington, DC: U.S. Department of Health and Human Services OoIG; 2020:1-41

57 Ze. ege. n EN, Yates AJ, Jevsevar DS. After the COVID-19 pandemic: returning to normalcy or returning to a new normal? J Arthroplasty 2020. Doi: 10.1016/j.arth.2020.04.040

58 Toy PC, Fournier MN, Throckmorton TW, Mihalko WM. Low rates of adverse events following ambulatory outpatient total hip arthroplasty at a free-standing surgery center. J Arthroplasty 2018;33(1):46-50

$59 \mathrm{~J}$, aib, aji M, Volpin A, Haddad FS, Konan S. Is outpatient arthroplasty safe? A systematic review. J Arthroplasty 2020. Doi:10.1016/j.arth.2020.02.022

60 Hoffmann JD, Kusnezov NA, Dunn JC, Zarkadis NJ, Goodman GP, Berger RA. The shift to same-day outpatient joint arthroplasty: a systematic review. J Arthroplasty 2018;33(4):1265-1274

61 Kogan M, Klein SE, Hannon CP, Nolte MT. Orthopaedic education during the COVID-19 pandemic. J Am Acad Orthop Surg 2020;28(11):e456-e464

62 Coe TM, Jogerst KM, Sell NM, et al. Practical techniques to adapt surgical resident education to the COVID-19 era. Ann Surg 2020. Doi:10.1097/SLA.0000000000003993 